\title{
14.
}

\section{Beiträge zur Theorie der elliptischen Functionen.}

\author{
(Von Herrn Dr. phil. G. Eisenstcin zu Berlin.)
}

I. Ableitung des biquadratischen Fundamentaltheorems aus der Theorie der Lemniscatenfunctionen, nebst Bemerkungen zu den Multiplications - und Transformationsformeln.

\section{1.}

$W_{\text {enn }} a+b i=m$ irgend eine ungerade complexe ganze Zahl vorstellt, und $a^{2}+b^{2}=p=N(m)$ gesetzt wird, so ist das Integral der Differenzialgleichung

$$
\frac{\partial y}{\sqrt{ }\left(1-y^{4}\right)}=(a+b i) \cdot \frac{\partial x}{\sqrt{ }\left(1-x^{4}\right)}
$$

wenn $y$ mit $x$ zuigleich verschwinden soll, von der Form:

$$
y=x \frac{A_{0}+A_{1} x^{4}+A_{2} x^{8}+\ldots+A_{k(p-1)} x^{p-1}}{1+B_{1} x^{4}+B_{2} x^{8}+\ldots .+B_{k(p-1)} x^{p-1}}=\frac{U}{V}
$$

wo die Coëfficienten $A_{0}, A_{1}$ etc. $B_{1}$ etc. ganze complexe Zahlen sind. In dieser Form des Integrals werden Zähler und Nenner keinen algebraischen und selbst keinen numerischen gemeinschaftichen Theiler haben. Es kommt darauf an, die noch unbekannten ganzen Coëfficienten zu finden, oder wenigstens diejenigen ihrer Eigenschaften zu entdecken, welche hier nöthig sein werden.

Man zeigt leicht, dafs $A_{0}=m$ ist; denn $A_{0}$ ist der Werth von $\frac{y}{x}$ für $x=0$, also $\boldsymbol{A}_{0}=\frac{\partial y}{\partial x}$ für $x=0$; nun verschwindet $y$ mit $x$ zugleich, also giebt die Differenzialgleichung (1.) für $x=0, \frac{\partial y}{\partial x}=m$.

Setzt man in (1.) und (2.) $x=\frac{1}{i^{\mu} \xi}, y=\frac{1}{\eta}$, und disponirt über die ganze Zahl $\mu$ auf passende Weise, so erhält man

$$
\begin{gathered}
\frac{\partial \eta}{\sqrt{\left(1-\eta^{4}\right)}}=m \frac{\partial \xi}{\sqrt{\left(1-\xi^{4}\right)}}, \\
\eta=i^{\mu} \xi \frac{\boldsymbol{B}_{z(p-1)}+\boldsymbol{B}_{\xi(p-5)} \xi^{4}+\ldots \ldots+\boldsymbol{B}_{1} \xi^{p-5}+\xi^{p-1}}{A_{k(p-1)}+A_{k(p-5)} \xi^{4}+\ldots .+A_{1} \xi^{p-5}+\xi^{p-1}}
\end{gathered}
$$

Crelle's Journal f. d. M. Bd. XXX. Heft 3. 
also ist auch

$$
\text { (3.) } y=i^{\mu} x \frac{\boldsymbol{B}_{\{(p-1)}+\ldots .+x^{p-1}}{A_{\dot{q}(p-1)}+\ldots .+A_{0} x^{p-1}}
$$

ein Integral der Differenzialgleichung (1.), und da dieses ebenfalls mit $x$ zugleich verschwindet, mithin mit (2.) identisch sein mufs, auch Zähler und Nenner keinen gemeinschaftlichen Theiler haben, so müssen Zähler und Nenner des neuen und des alten $y$ aus (3.) und aus (2.), abgesehen von einer complexen Einheit, einzeln verglichen mit einander übereinstimmen. Eine solche Vergleichung giebt:

$$
\text { (4.) } \quad A_{0}=i^{v} B_{i(p-1)}, A_{1}=i^{v} B_{k(p-5)}, \ldots A_{i(p-r)}=i^{v} B_{1}, \quad A_{k(p-1)}=i^{\nu}
$$

Der Exponent $\nu$ ist eine noch unbestimmte ganze Zahl. Um ihn zu bestimmen und die nachfolgenden Untersuchungen mit gröfserer Leichtigkeit zu führen, sei allgemein $\varphi(t)$ diejenige Function von $t$, welche mit $t$ zugleich verschwindet und der Differentialgleichung genügt:

$$
\frac{\partial \varphi(t)}{\partial t}=\sqrt{ }\left(1-\varphi(t)^{4}\right)
$$

Aufserdem setze man

$$
\omega=4 \int_{0}^{1} \frac{\partial z}{\sqrt{ }\left(1-z^{4}\right)}=\frac{\Gamma\left(\frac{1}{4}\right)^{2}}{\sqrt{(2 \pi)}}, \text { so dafs } \varphi\left(\frac{\omega}{4}\right)=1 \text { wird. }
$$

Die Function $\varphi(t)$ ist complex periodisch, und man hat $\varphi(t+k \omega)=\varphi(t)$, wenn $k$ irgend eine ganze complexe Zahl vorstellt. Die Fundamental-Eigenschaften der Function $\varphi$ sind durch die beiden Gleichungen gegeben:

$$
\begin{aligned}
\varphi(i t) & =i \varphi(t) \quad \text { und } \\
\varphi\left(t+t^{\prime}\right) & =\frac{\varphi(t) v\left(1-\varphi\left(t^{\prime}\right)^{4}\right)+\varphi\left(t^{\prime}\right) \sqrt[V]{ }\left(1-\varphi(t)^{4}\right)}{1+\varphi(t)^{2} \varphi\left(t^{\prime}\right)^{2}} .
\end{aligned}
$$

Hieraus und aus der erwähnten Periodicität, die sich aus den Fundamentalgleichungen unmittelbar ergiebt, leitet man leicht die Formel ab:

$$
\varphi\left[(2 \alpha+1+2 \beta i) \frac{\omega}{4}\right]=(-1)^{\alpha+\beta},
$$

wenn $\alpha$ und $\beta$ zwei reelle ganze Zahlen sind. Das Integral (2.) der Differenzialgleichung (1.) läfst sich jetzt in folgender Form schreiben:

$$
\varphi(m t)=\varphi(t) \cdot \frac{A_{0}+A_{1} \varphi(t)^{4}+\ldots .+A_{z}(p-1) \varphi(t)^{p-1}}{1+B_{1} \varphi(t)^{4}+\ldots .+B_{\tilde{z}}(p-1) \varphi(t)^{p-1}} .
$$

Um nun den Werth von $\nu$ in (4.) zu bestimmen, setze man statt $t$ den speciellen Werth $\frac{\omega}{4}$, so dafs $\varphi(t)=1$ wird: da für diesen Werth, wenn man noch $a$ ungerade $=2 \alpha+1, b$ gerade $=2 \beta$ annimmt, nach dem vorhin Be- 
merkten $\varphi(m t)=\varphi\left(m \cdot \frac{\omega}{4}\right)$ in $(-1)^{\alpha+\beta}$ übergeht, so erhält man aus der eben geschriebenen Formel

$$
(-1)^{\alpha+\beta}=\frac{A_{0}+A_{1}+\ldots .+A_{t(p-1)}}{1+B_{1}+\ldots .+B_{t(p-1)}}
$$

für einen Werth von $m$, dessen reeller Theil ungerade, Coëfficient von $i$ gerade ist, also kommt, wenn man für $\boldsymbol{A}_{0}, \boldsymbol{A}_{1}$ etc. die Werthe durch die $\boldsymbol{B}$ aus (4.) einsetzt:

$$
(-1)^{\alpha+\beta}=i^{\nu} \frac{B_{4(p-1)}+B_{\frac{4}{4}(p-5)}+\ldots .+B_{1}+1}{1+B_{1}+\ldots .+B_{4(p-5)}+B_{4(p-1)}}, \text { welches }=i^{\nu},
$$

weil Zähler und Nenner hier einander gleich sind und nicht zugleich verschwinden können; mithin $i^{v}=(-1)^{\alpha+\beta}$.

Für alle Fälle reicht es hin, $m$ primär, nach der von Gau/s diesem Worte beigelegten Bedeutung, d. h. $\equiv 1(\bmod .2+2 i)$ zu nehmen, denn alle möglichen complexen ungeraden Zahlen lassen sich aus den primären durch Multiplication mit complexen Einheiten $\pm 1, \pm i$ ableiten. Für einen primären Werth von $m$ ist $\alpha+\beta$ gerade, also $i^{\nu}=+1$, mithin werden die Multiplicationsformeln für einen primüren Werth von m, aber nur für einen solchen, von der Form:

$$
\begin{aligned}
\varphi(m t) & =\varphi(t) \frac{m+A_{1} \varphi(t)^{4}+\ldots+\varphi(t)^{p-1}}{1+A_{i(p-5)} \varphi(t)^{4}+\ldots+m \varphi(t)^{p-1}}, \\
y & =\frac{m x+A_{1} x^{5}+\ldots+x^{p}}{1+A_{4(p-5)} x^{4}+\ldots+m x^{p-1}}=\frac{U}{V} .
\end{aligned}
$$

Wir kommen jetzt zu einem Satze, welcher die Grundlage für alle Anwendungen der Lemniscatentheilung auf Zahlentheorie zu bilden scheint und welcher um so merkwürdiger ist, da er sich ganz einfach aus der Differenzialgleichung ableiten läfst. Es ist der folgende:

„Wenn $m$ eine zweigliedrige complexe Primzahl, also $p$ eine reelle Primzahl $\equiv 1(\bmod .4)$ ist, so sind alle Coëfficienten des Zählers $U$ bis auf den letzten, so wie alle Coëfficienten des Nenners $\boldsymbol{V}$ bis auf den ersten durch $m$ theilbar." so kommt

In der That, setzt man $y=\frac{U}{V}$ in die Differenzialgleichung (1.) ein,

$$
\boldsymbol{V} \frac{\partial U}{\partial x}-\boldsymbol{U} \frac{\partial \boldsymbol{V}}{\partial x}=m \cdot \frac{\sqrt[V]{ }\left(\boldsymbol{V}^{4}-U^{4}\right)}{\sqrt{ }\left(1-x^{4}\right)}
$$

Hieraus folgt einerseits, dafs der Ausdruck $\frac{\sqrt{ }\left(V^{4}-U^{4}\right)}{\sqrt{\left(1-x^{4}\right)}}$ einer ganzen Function von $x^{4}$ gleich ist, welche wir durch $\boldsymbol{T}$ bezeichnen wollen. Andrerseits ist $V^{4}-U^{4}$ eine ganze Function von $x^{4}$ mit ganzen complexen Coèfficienten, 
deren constantes Glied $=1$ ist, und welche für $x^{4}=1$ verschwindet, also ist $V^{4}-U^{4}$ durch $1-x^{4}$ theilbar und $\frac{V^{4}-U^{4}}{1-x^{4}}=T^{2}$ eine ganze Function von $x^{4}$ mit ganzen Coëfficienten, deren constantes Glied (erster Coëfficient) $=1$; mithin mufs auch $\boldsymbol{T}$ selbst, welches schon als ganze Function erkannt wurde, lauter ganze Coëfficienten haben. Da nun

war, so folgt hieraus

$$
\boldsymbol{V} \frac{\partial U}{\partial x}-\boldsymbol{U} \frac{\partial \boldsymbol{V}}{\partial x}=m \boldsymbol{T}
$$

$$
\boldsymbol{V} \frac{\partial \boldsymbol{U}}{\partial x}-\boldsymbol{U} \frac{\partial \boldsymbol{V}}{\partial \boldsymbol{x}} \equiv 0(\bmod . m)
$$

eine Congruenz, welche in dem Sinne zu verstehen, dafs jeder Coëfficient in der nach Potenzen von $x$ geordneten linken Seite durch $m$ theilbar ist. Entwickelt man wirklich, nach den Formeln

$$
\begin{aligned}
& \frac{\partial U}{\partial x}=A_{0}+5 A_{1} x^{4}+9 A_{2} x^{3}+13 A_{3} x^{12}+\ldots \\
& \frac{\partial V}{\partial x}=4 B_{1} x^{3}+8 B_{2} x^{7}+12 B_{3} x^{11}+\ldots
\end{aligned}
$$

so kommt

$$
\begin{aligned}
\boldsymbol{V} \frac{\partial \boldsymbol{U}}{\partial x}-\boldsymbol{U} \frac{\partial \boldsymbol{V}}{\partial x} & =\boldsymbol{A}_{0}+\left(-3 \boldsymbol{A}_{0} \boldsymbol{B}_{1}+5 \boldsymbol{A}_{1}\right) \boldsymbol{x}^{4}+\left(-7 \boldsymbol{A}_{0} \boldsymbol{B}_{2}+\boldsymbol{A}_{1} \boldsymbol{B}_{1}+9 \boldsymbol{A}_{2}\right) \boldsymbol{x}^{8} \\
& +\left(-11 \boldsymbol{A}_{0} \boldsymbol{B}_{3}-3 \boldsymbol{A}_{1} \boldsymbol{B}_{2}+5 \boldsymbol{A}_{2} \boldsymbol{B}_{1}+13 \boldsymbol{A}_{3}\right) \boldsymbol{x}^{12}+\text { etc. }
\end{aligned}
$$

Da nun hier jeder einzelne Coëfficient durch $m$ theilbar sein soll, und für eine zweigliedrige Primzahl $m$ die numerischen Factoren $5,9,13, \ldots, p-4$ nicht durch $m$ aufgehen, so ersieht man, wenn die schon gewonnenen Congruenzen bei jeder folgenden mitbenutzt werden, dafs $\boldsymbol{A}_{0}, \boldsymbol{A}_{1}, \boldsymbol{A}_{2}, \ldots \boldsymbol{A}_{\frac{1}{4}(p-5)}$ nothwendig durch on theilbar sein müssen. Z. B. $\boldsymbol{A}_{0}$ so wie $-3 \boldsymbol{A}_{0} \boldsymbol{B}_{\mathbf{1}}+5 \boldsymbol{A}_{1}$ sind durch $m$ theilbar, also auch $5 \boldsymbol{A}_{1}$; aber 5 ist nicht durch $m$ theilbar, also mufs es $A_{1}$ sein; ferner geht $m$ in $-\tau A_{0} B_{2}+A_{1} B_{1}+9 A_{2}$ auf, aber auch in $A_{0}$ und $A_{1}$, wie eben gefunden, mithin auch in $9 A_{2}$, also in $A_{2}$, da 9 nicht durch $m$ theilbar, und in derselben Weise fort. Auf den letzten Coëfficienten $\boldsymbol{A}_{\dot{\mathfrak{d}}(p-1)}$ läfst sich diese Art zu schliefsen nicht mehr anwenden, da sehr wohl $\boldsymbol{p} \boldsymbol{A}_{\frac{1}{\mathbf{4}(p-1)}}$ durch $\boldsymbol{m}$ theilbar sein kann und sein mufs, ohne dafs $\boldsymbol{A}_{\frac{1}{\mathbf{4}(p-1)}}$ es zu sein braucht. Ebenso wenig kann man diese Schlüsse anwenden, wenn $m$ eine eingliedrige Primzahl ist: in diesem Falle werden mehrere der Zahlen

durch $m$ theilbar.

$$
5,9,13, \ldots, p-4
$$


11. Eisenstein, zur Theorie der Lemniscatenfunct. und Transformation. 189

Wir sehen also: „dafs für jede primäre zweigliedrige complexe Prim„zahl $m \varphi(m t)$ sich auf die Form bringen lăfst:

$$
\varphi(m t)=\varphi(t) \cdot \frac{m £+\varphi(t)^{p-1}}{1+m \mathbb{E}},
$$

"wo und $\mathscr{G}$ ganze Functionen von $\varphi(t)^{4}$ mit ganzen complexen Coëffi"cienten sind."

Beispiele. Für $m=-1+2 i, p=5$ wird $y=\frac{(-1+2 i) x+x^{5}}{1+(-1+2 i) x^{4}}$; für $m=3+2 i, p=13$ wird

$$
y=\frac{(3+2 i) x+(7-4 i) x^{3}+(-11+10 i) x^{0}+x^{13}}{1+(-11+10 i) x^{4}+(7-4 i) x^{8}+(3+2 i) x^{12}}
$$

und man hat

$$
7-4 i=(3+2 i)(1-2 i), \quad-11+10 i=(3+2 i)(-1+4 i),
$$

so dafs

$$
\begin{aligned}
\mathfrak{f} & =1+(1-2 i) x^{4}+(-1+4 i) x^{8}, \\
\mathfrak{G} & =(-1+4 i) x^{4}+(1-2 i) x^{8}+x^{12} .
\end{aligned}
$$

Für $m=1+4 i, p=17$ sind die Coëfficienten des Zählers $U$ nach der Reihe:

$$
\begin{gathered}
1+4 i,-20-12 i=(1+4 i)(-4+4 i),-10+28 i=(1+4 i)(6+4 i), \\
12-20 i=(1+4 i)(-4-4 i), 1 .
\end{gathered}
$$

Um auch davon ein Beispiel zu geben, dafs der Satz für Nichtprimzahlen seine Gültigkeit verliert, betrachte man die Coëfficienten des Zählers für $m=5$, $p=25$, welche

$$
5,-62,-105,300,-125,50,1
$$

sind, wo -62 nicht durch 5 theilbar ist.

2.

Ein complexes Restensystem für den (ungeraden, primären) Modul $m$ läfst sich, nach Ausschliefsung des durch den Modul theilbaren Gliedes, auf viele Arten folgendermafsen gruppiren:

$$
\text { (7.) }\left\{\begin{array}{l|l|l|l}
r_{1} & i r_{1} & -r_{1} & -i r_{1} \\
r_{2} & i r_{2} & -r_{2} & -i r_{2} \\
r_{3} & i r_{3} & -r_{3} & -i r_{3} \\
\vdots & \vdots & \vdots & \vdots \\
r_{\frac{1}{4}(p-1)} & i r_{\frac{1}{4}(p-1)} & -r_{\frac{1}{4}(p-1)} & -i r_{\frac{1}{4}(p-1)}
\end{array}\right.
$$

Nimmt man nämlich für $\boldsymbol{r}_{1}$ irgend ein Glied des Restensystems, so sind die vier Zahlen der ersten Horizontalreihe des Schemas unter einander incongruent; erschöpfen sie das Restensystem noch nicht, so nehme man für $\boldsymbol{r}_{2}$ irgend eines 
der noch übrig bleibenden Glieder, worauf dann die vier Glieder der zweiten Horizontalreihe unter einander and $z u$ denen der ersten incongruent sein werden; und so weiter fort, bis alle Glieder des Restensystems erschöpft sind *). Wir nennen diese Gruppirung: die Eintheilung eines Restensystems in vier associirte Theile, und jeden dieser vier Theile kurz ein Viertel-Restensystem. Nachdem man auf irgend eine Art diese Eintheilung vorgenommen hat, kann man alle übrigen Arten daraus ableiten, indem man die Glieder jeder einzelnen Horizontalreihe cyklisch permutirt, so dafs die Anzahl der Arten $=4^{\frac{1}{4}(p-1)}$ wird. Der zuerst von Gau/s angegebene Gedanke, ein Restensystem in dieser Weise zu theilen, ist ebenso wichtig, als der Begriff der Congruenz selbst, und beruht auf der Betrachtung solcher Zahlen, welche sich zu associirten ebenso verhalten, wie congruente Zahlen zu den gleichen. Mit zwei Worten gesagt, ist ein Viertelrestensystem jede Reihe von $\frac{1}{4}(p-1)$ Zahlen, aus welcher man durch Addition von Vielfachen des Moduls und durch Multiplication mit complexen Einheiten alle nicht durch den Modul theilbaren Zahlen, und jede nur einmal erzeugen kann.

Nach dieser Vorbemerkung wenden wir uns zu der Betrachtung der Wurzeln der Gleichung $\frac{U}{x}=0 . \quad \frac{U}{x}$ ist eine ganze Function von $x^{4}$ vom $\frac{1}{4}(p-1)$ ten Grade, also hat die Gleichung $\frac{U}{x}=0$, wenn man in ihr $x^{4}=z$ als den Unbekannten betrachtet, d. h. die Gleichung

$$
\mathbf{z}^{\frac{1}{4}(p-1)}+A_{\frac{1}{4}(p-5)} z^{\frac{1}{4}(p-1)-1}+\ldots+A_{1} z+m=0,
$$

$\frac{1}{4}(p-1)$ Wurzeln $\approx$, welche, da die Gleichung (8.) folgende $\varphi(m t)=0$ nach sich zieht, offenbar durch die Formel $z=\varphi\left(\frac{r \omega}{m}\right)^{4}$ gegeben sind, in der $\boldsymbol{r}$ den Inbegriff der Zahlen $\boldsymbol{r}_{1}, \boldsymbol{r}_{2}, \ldots, \boldsymbol{r}_{\frac{1}{4}(p-1)}$ aus der ersten Verticalcolumne des Schemas (7.) bezeichnet. Da die Coëfficienten der Gleichung (8.) ganz sind, so ist auch jede symmetrische ganze Function ihrer Wurzeln also "jede symmetrische Function der $\frac{1}{4}(p-1)$ Gröfsen

$$
\varphi\left(\frac{r_{1} \omega}{m}\right)^{4}, \varphi\left(\frac{r_{2} \omega}{m}\right)^{4}, \varphi\left(\frac{r_{3} \omega}{m}\right)^{4}, \text { etc. }
$$

*) Beiläufig ergiebt sich hieraus, dals die Norm einer complexen Zahl aus 4ten Wurzeln der Einheit $\equiv 1$ (mod. 4) ist; in dem speciellen Falle für 4te Wurzeln der Einheit ist zwar diese Bemerkung von wenig Belang, aber interessant ist, dafs sich ein analoger Schlufs bei complexen Zahlen aus beliebig hohen Wurzeln der Einheit anwenden läßst. 
"deren Inbegriff durch $\varphi\left(\frac{r \omega}{m}\right)^{4}$ bezeichnet wird, eine ganze complexe Zahl," und da das Product aller Wurzeln dem positiven oder negativen letzten Coëfficienten gleich ist, je nachdem der Grad der Gleichung eine gerade oder ungerade Zahl ist, so hat man namentlich

$$
\begin{aligned}
& \text { (9.) } \quad \Pi \varphi\left(\frac{r \omega}{m}\right)^{4}=(-1)^{\frac{1}{4}(p-1)} \cdot m \text {, oder } \\
& \left(9^{\prime} .\right) \quad \sqrt{ } /\left((-1)^{\frac{1}{4}(p-1)} \cdot m\right)=\Pi \varphi\left(\frac{r \omega}{m}\right),
\end{aligned}
$$

wo sich das Multiplicationszeichen $\Pi$ auf alle Werthe von $r$ bezieht. Die Wichtigkeit der Formel $\left(9^{\prime}\right.$.) besteht darin, dafs sie die einzige ist, durch welche man bis jetzt die vierte Wurzel aus einer complexen Zahl so zu sagen rational ausdrücken kann; sie vertritt zum Theil die Stelle der Gau/sischen Formeln in der complexen Theorie. - Das hier Gesagte gilt übrigens, m mag eingliedrig oder zweigliedrig sein, wenn nur primăr.

3.

Um zu den biquadratischen Resten überzugehen, sei, mit Beibehalten der bisherigen Bezeichnungen, $m$ eine beliebige primäre Primzahl, gleichviel ob eingliedrig oder zweigliedrig, $n$ dagegen eine primäre und zweigliedrige Primzahl, die von $m$ verschieden ist; $p$ und $q$ seien respective die Normen von $m$ und $n$. - Multiplicirt man alle $r$ mit $n$, so befinden sich die Reste der verschiedenen Producte $n r$ theils unter den $r$, theils unter den $i r,-r$, oder -ir; man kann also allgemein setzen

$$
n \boldsymbol{r} \equiv \boldsymbol{i}^{\mu} \boldsymbol{r}^{\prime}(\bmod . m),
$$

wo sich die verschiedenen $\boldsymbol{r}^{\prime}$, die den verschiedenen $\boldsymbol{r}$ entsprechen, alle unter den $\boldsymbol{r}$ befinden, und sämmtlich von einander verschieden sind ${ }^{*}$ ), also, wenn auch in anderer Ordnung, mit allen $\boldsymbol{r}$ zusammenfallen. Aus der eben geschriebenen Congruenz folgt wegen der complexen Periodicität der Function $\varphi$ und wegen $\varphi(i t)=i \varphi(t)$ :

$$
\varphi\left(\frac{n r \omega}{m}\right)=\varphi\left(\frac{i^{\mu} r^{\prime} \omega}{m}\right)=i^{\mu} \varphi\left(\frac{r^{\prime} \omega}{m}\right), \quad i^{\mu}=\frac{\varphi\left(\frac{n r \omega}{m}\right)}{\varphi\left(\frac{r^{\prime} \omega}{m}\right)}
$$

*) Weil nicht $u r_{\sigma} \equiv \pm n r_{\tau}$ oder $\equiv \pm i n r_{\tau}$ sein kann, ohne dafs $r_{\sigma} \equiv \pm r_{\tau}$ odér $\equiv \pm i r_{\tau}$ wäre, welches letztere aber der Constructionsweise unseres Schemas (7.) offonbar widerspricht. 
mithin erhält man, wenn dieser Werth von $i^{\mu}$ in die Congruenz eingesetzt wird, in allen Fällen :

$$
n r \equiv r^{\prime} \cdot \frac{\varphi\left(\frac{\| r \omega}{m}\right)}{\varphi\left(\frac{r^{\prime} \omega}{m}\right)}(\bmod . m)
$$

und da alle $\boldsymbol{r}^{\prime}$, wenn auch in anderer Ordnung, mit allen $\boldsymbol{r}$ zusammenfallen, so kommt durch Multiplication über alle $r$ :

$$
n^{\frac{1}{4}(p-1)} \Pi(r) \equiv \Pi(r) \frac{\Pi \varphi(n t)}{\Pi \varphi(t)}(\bmod . m),
$$

wo zur Erleichterung des Druckes $t$ statt $\frac{r \omega}{m}$ geschrieben ist. Da nun keine der Zahlen $r$ durch $m$ theilbar ist, so kann man auf beiden Seiten mit $\Pi(r)$ fortdividiren und erhält

$$
\text { (10.) } n^{\frac{2}{4}(p-1)} \equiv \frac{\Pi_{\varphi(n t)}}{\Pi \varphi(t)}(\bmod . m),
$$

was auch so geschrieben werden kann:

$$
\text { (10'.) }\left[\frac{n}{m}\right]=\Pi \frac{\varphi(n t)}{\varphi(t)},
$$

sobald man durch $\left[\frac{n}{m}\right]$ diejenige complexe Einheit bezeichnet, welcher die Potenz $n^{\frac{1}{4}(p-1)}(\bmod . m)$ congruent ist.

Zur weitern Behandlung der'in $\left(10^{\prime}\right.$.) für $\left[\frac{n}{m}\right]$ aufgestellten analytischen Formel dient das in \$. 1. gefundene Resultat, nach welchem

$$
\frac{\varphi(n t)}{\varphi(t)}=\frac{n F\left[\varphi(t)^{4}\right]+\varphi(t)^{q-1}}{1+n G\left[\varphi(t)^{4}\right]},
$$

weil $\boldsymbol{n}$ als zweigliedrige primäre Primzahl angenommen worden ist; $\boldsymbol{F}$ und $\boldsymbol{G}$ sind ganze Functionen von $\varphi(t)^{4}$ mit ganzen complexen Coëfficienten. Hiernach kommt wegen $\left(10^{\prime}\right.$.)

$$
\left[\frac{n}{m}\right] \cdot \Pi\left\{1+n G\left[\varphi\left(\frac{r \omega}{m}\right)^{4}\right]\right\}=\Pi\left\{n \boldsymbol{F}\left[\varphi\left(\frac{r \omega}{m}\right)^{4}\right]+\varphi\left(\frac{r \omega}{m}\right)^{q-1}\right\} .
$$

Die beiden Producte zur Linken und zur Rechten in dieser Formel, in denen sich die Multiplication auf alle Werthe von $r$ bezieht, lassen sich, wenn man die Multiplication ausführt, rein algebraisch nach Potenzen von $n$ ordnen, und man sieht, dafs dann jeder Coëfficient dieser Entwickelungen eine symmetrische Function der Größsen $\varphi\left(\frac{r \omega}{m}\right)^{4}$, also nach $\$$. 2. einer ganzen complexen Zahl 
14. Eisenstein, zur Theorie der Lemniscatenfunct. und Transformation. 193

gleich wird: so dafs man erhält

$$
\begin{aligned}
& {\left[\frac{n}{m}\right] \cdot\left\{1+a_{1} n+a_{2} n^{2}+\ldots+a_{\frac{1}{4}(p-1)} n^{\frac{1}{4}(p-1}\right\}=} \\
& \Pi \varphi\left(\frac{r \omega}{m}\right)^{q-1}+b_{1} n+b_{2} n^{2}+\ldots .+b_{\frac{1}{4}(p-1)} n^{\frac{1}{4}(p-1)},
\end{aligned}
$$

wo $a_{1}, a_{2}$ etc., $b_{1}, b_{2}$ etc. sämmtlich ganze complexe Zablen, nämlich sämmtlich symmetrische ganze Functionen der Wurzeln der Gleichung (8.) sind. Setzt man hier für $\Pi \varphi\left(\frac{r \omega}{m}\right)^{q-1}$ den Werth $\left[(-1)^{\frac{1}{4}(p-1)} m\right]^{\frac{1}{4}(q-1)}$ aus (9.) und läfst auf beiden Seiten die durch $n$ theilbaren Glieder fort, so steht man am Ziele, denn man bekommt:

$$
\begin{aligned}
{\left[\frac{n}{m}\right] } & \equiv(-1)^{\frac{1}{4}(p-1) \cdot \frac{1}{4}(q-1)} \cdot m^{\frac{1}{4}(q-1)}(\bmod . n), \text { d. h. } \\
\text { (12.) }\left[\frac{n}{m}\right] & =(-1)^{\frac{1}{4}(p-1) \cdot \frac{1}{4}(q-1)} \cdot\left[\frac{m}{n}\right] .
\end{aligned}
$$

Dies ist das Gau/sische Reciprocitätsgesetz für alle Fälle mit Ausnahme des einzigen, wenn beide Zahlen reell (eingliedrig) sind. In diesem letzteren Falle folgt der Satz unmittelbar ohne Weiteres aus dem Fermatschen Satze; denn $\operatorname{sind} m$ und $n$ beide reelle Primzahlen $\equiv 3(\bmod .4)$, so sind $m^{2}$ und $n^{2}$ ihre Normen und man hat

$$
\begin{aligned}
& ( \pm m)^{\frac{1}{4}\left(n^{2}-1\right)}=\left( \pm m^{\frac{1}{4}(n+1)}\right)^{n-1} \equiv 1(\bmod . n), \text { und } \\
& ( \pm n)^{\frac{1}{4}\left(m^{2}-1\right)}=\left( \pm n^{\frac{1}{4}(m+1)}\right)^{m-1} \equiv 1(\bmod . m), \text { also }\left[\frac{m}{n}\right]=\left[\frac{n}{m}\right] .
\end{aligned}
$$

Um dem Reciprocitätssatze (12.) die Form zu geben, in welcher Gau/s ihn dargestellt hat, darf man nur bemerken, dafs für $m=a+b i, p=a^{2}+b^{2}$, $\frac{1}{4}(p-1)=\frac{1}{4}\left(a^{2}-1\right)+\frac{1}{4} b^{2}=\frac{1}{4}\left(a^{2}-1\right)+\left(\frac{1}{2} b\right)^{2}$ wird, und dafs $a^{2}$ als Quadrat einer ungeraden Zahl $\equiv 1(\bmod .8)$, mithin $\frac{1}{4}\left(a^{2}-1\right)$ gerade ist, also $\frac{1}{4}(p-1) \equiv$ $\left(\frac{1}{2} b\right)^{2} \equiv \frac{1}{2} b(\bmod .2)$ wird. Da $a+b i$ primär ist, so hat man $\frac{1}{2} b \equiv \frac{1}{2}(a-1)$ (mod.2), also auch $\frac{1}{4}(p-1) \equiv \frac{1}{2}(a-1)(\bmod .2)$; setzt man ebenso $n=c \nmid d i$, so ist auf dieselbe Weise $\frac{1}{4}(q-1) \equiv \frac{1}{2}(c-1)(\bmod .2)$, also kann man in (12.) statt $\frac{1}{4}(p-1) \cdot \frac{1}{4}(q-1)$ auch $\frac{1}{2}(a-1) \cdot \frac{1}{2}(c-1)$ schreiben; man erhält so

$$
\left[\frac{n}{m}\right]=(-1)^{\ddagger(a-1) \cdot t(c-1)} \cdot\left[\frac{m}{n}\right]
$$

und dies ist die Gau/sische Form des Satzes.

Zu bemerken ist noch, dafs aus der Verbindung der Formeln ( $\boldsymbol{9}^{\prime}$.) und $\left(10^{\prime}\right.$.)

Crelle's Journal f. d. M. Bd. XXX. Heft 3. 


$$
\begin{aligned}
& \Pi \varphi\left(\frac{r \omega}{m}\right)=J^{4}\left((-1)^{\frac{1}{4}(p-1)} m\right), \\
& \Pi \varphi\left(\frac{n r \omega}{m}\right)=\left[\frac{n}{m}\right] \cdot \Pi \varphi\left(\frac{r \omega}{m}\right), \text { die folgende hervorgeht } \\
& \Pi \varphi\left(\frac{n r \omega}{m}\right)=\left[\frac{n}{m}\right]^{4} /\left((-1)^{\frac{1}{4}(p-1)} m\right) .
\end{aligned}
$$

Die erste dieser drei zuletzt geschriebenen Formeln gilt für ein beliebiges primäres $m$, die beiden übrigen dagegen nur für Primzahlen $m$; $n$ ist ganz beliebig, nur nicht durch $\boldsymbol{n}$ theilbar; $\boldsymbol{r}$ durchläuft bei der Multiplication ein Viertelrestensystem $(\bmod . m)$.

4.

Es möchte hier nicht am unrechten Platze sein, eine einfache Methode anzugeben, nach welcher man die Coëfficienten in dem Zähler und Nenner der Multiplicationsformeln für die Lemniscate berechnen kann. - Die complexe ganze Zahl $m$ wird hier nur der einzigen Beschränkung unterworfen, primär, d. h. $\equiv 1(\bmod 2+2 i)$ zu sein.

Sei

$$
\text { (1.) } \frac{\partial x}{\partial t}=\sqrt{ }\left(1-x^{4}\right) \text { und } \frac{\partial y}{\partial t}=m \sqrt{ }\left(1-y^{4}\right) \text {, }
$$

welche beiden Gleichungen die Stelle der einzigen $\frac{\partial y}{\partial x}=\frac{m \sqrt{ }\left(1-y^{4}\right)}{\sqrt{ }\left(1-x^{4}\right)}$ vertreten, so ist

$$
y=\frac{U}{V}=\frac{x^{p}+B_{1} x^{p-4}+\ldots+A_{1} x^{5}+m x}{m x^{p-1}+A_{1}} \frac{x^{p-5}+\ldots+B_{1} x^{4}+1}{1} .
$$

Betrachtet man in der Gleichung

$$
U-V y=0, \text { d. h. }
$$

$$
x^{p}-m y \cdot x^{p-1}+B_{1} x^{p-4}-A_{1} y \cdot x^{p-5}+\ldots+m x-y=0
$$

$x$ als Function von $y$, so geben die $p$ Wurzeln dieser Gleichnng, welche wir durch

$$
\text { (3.) } x_{1}, x_{2}, x_{3}, \ldots x_{p}
$$

bezeichnen, $\boldsymbol{p}$ Functionen von $y$, welche sämmllich den Differenzialgleichungen (1.) genügen. Jede symmetrische Function der Gröfsen $x_{1}, \dot{x}_{2}$ etc. läfst sich durch die Coëfficienten der Gleichung (2.) ausdrücken, und könnte man umgekehrt jede symmetrische Function dieser Gröfsen auf irgend einem Wege direct bestimmen, so hätte man auch die Coëfficienten der Gleichung (2.), also auch die Coëfficienten des Zählers und Nenners $\boldsymbol{A}_{\mathbf{1}}$ etc. $\boldsymbol{B}_{\mathbf{1}}$ etc. 
Da in der Gleichung (2.) der Coëfficient von $x^{p-1}$ gleich - my, der von $x^{p-2}$ Null ist, so ist die Summe der Gröfsen (3.) $=m y$, die Summe ihrer Combinationen zu zweien $=0$, folglich auch die Summe ihrer Quadrate $=m^{2} y^{2}$; dieses sehr bekannte Resultat mag so geschrieben werden

$$
\text { (4.) } \Sigma x=m y, \quad \Sigma x^{2}=m^{2} y^{2} \text {, }
$$

indem allgemein durch $\Sigma \boldsymbol{H}^{\prime}(\boldsymbol{x})$ die Summe

bezeichnet wird.

$$
\boldsymbol{F}\left(\boldsymbol{x}_{1}\right)+\boldsymbol{F}\left(\boldsymbol{x}_{2}\right)+\ldots+\boldsymbol{F}\left(\boldsymbol{x}_{p}\right)
$$

Durch fortgesetztes Differenziiren der (4.) wird es gelingen, alle symmetrischen Verbindungen der Gröfsen (3.) zu bestimmen; da sich indessen alle symmetrischen Verbindungen in die Polenzsummen ausdrücken lassen, so genügt es, diese letzteren zu finden; es seien die Potenzsummen der Gröfsen (3.) durch $\boldsymbol{S}_{0}, \boldsymbol{S}_{1}, \boldsymbol{S}_{2}$ etc. bezeichnet, so dafs

$$
\mathbf{S}_{\mu}=\Sigma x^{\mu}=x_{1}^{\mu}+x_{2}^{\mu}+\ldots+x_{p}^{\mu}
$$

$\mathbf{S}_{0}$ ist $=1+1+\ldots+1=p$.

In der Trigonometrie besteht eines der wichtigsten Principien, durch welches man zu linearen Ausdrücken gelangt; in der Entwicklung der Potenzen von Kreisfunctionen nach Sinus oder Cosinus vielfacher Bogen; da dieses Hülfsmittel bei den elliptischen Functionen fehlt, so hat Jacobi in seinen Fundamenten ein anderes angegeben; welches sich auf die Möglichkeit gründet, die höheren Differenzialquotienten elliptischer Functionen durch die Potenzen dieser Functionen, und umgekehrt die Potenzen durch die Differenzialquotienten linear auszudrücken. Wie mancher schöne mathematische Gedanke wegen Mangel an analytischen Arbeitskräften unfruchtbar bleibt, so scheint auch dieses Princip, aufser der von Jacobi gemachten Anwendung, nicht weiter benutzt worden zu sein. Bei unserem Probleme hier kann das eben erwähnte Hülfsmittel mit Erfolg angewandt werden. Differenziiren wir nămlich die Gleichungen (4.) $\mu$ mal nach $t$, so dafs wir erhalten

$$
\text { (5.) } \quad \Sigma \frac{\partial^{\mu} x}{\partial t^{\mu}}=m \frac{\partial^{\mu} y}{\partial t^{\mu}}, \quad \sum \frac{\partial^{\mu}\left(x^{2}\right)}{\partial t^{\mu}}=m^{2} \frac{\partial^{\mu}\left(y^{2}\right)}{\partial t^{\mu}},
$$

verwandeln für gerade Werthe von $\mu$ die Differenzialquotienten rechts vom Gleichheitszeichen in Potenzen von $y$ und setzen dann die Summen links in die Formeln ein, welche diePotenzen von $x$ in die Differenzialquotienten von $x$ oder $x^{2}$, also auch die Potenzsummen $\Sigma x^{\mu}$ in die Summen von Differenzialquotienten ausdrücken, so erhalten wir direct die sämmtlichen Potenzsummen $\mathbb{S}_{\mu}$ als Punctio- 
nen von $y$. Setzt man sodann $y=0$, so bekommt man hieraus die Potenzsummen derjenigen Gröfsen, welche den Zähler der Multiplicationsformel verschwinden machen.

In der That, differenziirt man die Gleichung $\frac{\partial x}{\partial t}=\sqrt{ }\left(1-x^{4}\right)$ einmal, 3 mal, 5 mal u. s. w. nach $t$, so kommt

$$
\begin{aligned}
& \frac{\partial^{2} x}{\partial t^{2}}=-2 x^{3}, \quad \frac{\partial^{4} x}{\partial t^{4}}=-12 x+24 x^{5}, \\
& \frac{\partial^{6} x}{\partial t^{6}}=72 \cdot\left(7 x^{3}-10 x^{7}\right), \text { u.s.w. }
\end{aligned}
$$

Ferner erbält man durch Differenziation der Gleichung $\frac{\partial\left(x^{2}\right)}{\partial t}=2 x \sqrt{ }\left(1-x^{4}\right)$ :

$$
\begin{aligned}
& \frac{\partial^{2}\left(x^{2}\right)}{\partial t^{2}}=2-6 x^{4}, \quad \frac{\partial^{4}\left(x^{2}\right)}{\partial t^{4}}=24\left(-3 x^{2}+5 x^{6}\right), \\
& \frac{\partial^{6}\left(x^{2}\right)}{\partial t^{6}}=144\left(-1+28 x^{4}-35 x^{8}\right), \quad \text { u. s. w. }
\end{aligned}
$$

Man kann jede folgende Gleichung aus der vorhergehenden ableiten mit Hülfe der Formel

$$
\frac{\partial^{2} \psi}{\partial t^{2}}=\left(1-x^{4}\right) \cdot \frac{\partial^{2} \psi}{\partial x^{2}}-2 x^{3} \cdot \frac{\partial \psi}{\partial x} .
$$

Die geraden Differenzialquotienten von $x, x^{2}$ nach $t$ werden also resp. durch ungerade, gerade ganze Functionen von $\boldsymbol{x}$ ausgedrückt; die Gleichungen, welche dies bewirken, seien der Kürze halber durch ( $\boldsymbol{E}_{\text {.) }}$ bezeichnet.

Dieselbe Behandlung der Gleichung $\frac{\partial y}{\partial t}=m \sqrt{ }\left(1-y^{4}\right)$ liefert

$$
\left(F^{*}\right) \quad\left\{\begin{array}{l}
\frac{\partial^{2} y}{\partial t^{2}}=-2 m^{2} y^{3}, \quad \frac{\partial^{4} y}{\partial t^{4}}=12 m^{4}\left(-y+2 y^{5}\right), \\
\frac{\partial^{6} y}{\partial t^{6}}=72 m^{6}\left(7 y^{3}-10 y^{7}\right), \quad \text { u. s. w. } \\
\frac{\partial^{2}\left(y^{2}\right)}{\partial t^{2}}=2 m^{2}\left(1-3 y^{4}\right), \quad \frac{\partial^{4}\left(y^{2}\right)}{\partial t^{4}}=24 m^{4}\left(-3 y^{2}+5 y^{6}\right), \\
\frac{\partial^{6}\left(y^{2}\right)}{\partial t^{6}}=144 m^{6}\left(-1+28 y^{4}-35 y^{8}\right), \text { u. s. w. }
\end{array}\right.
$$

Diese Gleichungen, welche wir durch $(F$.) bezeichnen, geben die geraden Differenzialquotienten von $y$ und $y^{2}$ nach $t$ durch ungerade, resp. gerade ganze Functionen von $\boldsymbol{y}$, und werden mit Hülfe der Formel

aus einander abgeleitet.

$$
\frac{\partial^{2} \psi}{\partial t^{2}}=m^{2}\left(1-y^{4}\right) \frac{\partial^{2} \psi}{\partial y^{2}}-2 m^{2} y^{3} \frac{\partial \psi}{\partial y}
$$


Die Gleichungen $\left(E\right.$.) lassen sich nach $x^{3}, x^{5}$, u. s. w., $x^{4}, x^{6}$, u. s. w. auflösen, und geben dann die ungeraden Potenzen von $x$ in die Differenzialquotienten von $x$, die geraden Potenzen von $x$ in die Differenzialquotienten von $x^{2}$ ausgedrückt, nämlich :

$$
\text { (G.) } \begin{cases}x^{3}=-\frac{1}{2} \frac{\partial^{2} x}{\partial t^{2}}, & x^{5}=\frac{1}{2} x+\frac{1}{24} \frac{\partial^{4} x}{\partial t^{4}}, \text { u. s. w. } \\ x^{4}=\frac{1}{3}-\frac{1}{6} \frac{\partial^{2}\left(x^{2}\right)}{\partial t^{2}}, & x^{6}=\frac{3}{5} x^{2}+\frac{1}{20} \frac{\partial^{4}\left(x^{2}\right)}{\partial t^{4}}, \text { u. s. w. }\end{cases}
$$

Am bequemsten lassen sich die Gleichungen (G.) nach der Formel

$$
x^{\mu}=\frac{\mu-3}{\mu-1} x^{\mu-4}-\frac{1}{(\mu-1)(\mu-2)} \frac{\partial^{2}\left(x^{\mu-2}\right)}{\partial t^{2}}
$$

jede aus den beiden vorhergehenden bilden. Diese Formel liefert die Gleichungen (G.) mit der gröfsten Leichtigkeit in beliebiger Menge, denn nach ihr darf man nur, um $x^{\mu}$ zu bekommen, in dem Ausdrucke für $x^{\mu-2}$ alle Differenziations-Exponenten um 2 Einheiten erhöhen und das $\frac{1}{(\mu-1)(\mu-2)}$ fache dieses so erhaltenen Ausdruckes von dem $\frac{\mu-3}{\mu-1}$ fachen des Ausdruckes für $x^{\mu-4}$ subtrahiren.

Setzt man in (G.) für $x$ seine Werthe $x_{1}, x_{2} \ldots x_{p}$ und summirt, so erhält man die Gleichungen $(\boldsymbol{H}$.)

$$
\begin{cases}\Sigma x^{3}=-\frac{1}{2} \Sigma \frac{\partial^{2} x}{\partial t^{2}}, & \Sigma x^{5}=\frac{1}{2} \Sigma x+\frac{1}{24} \Sigma \frac{\partial^{4} x}{\partial t^{4}}, \text { u.s. w. } \\ \Sigma x^{4}=\frac{1}{3} p-\frac{1}{6} \Sigma \frac{\partial^{2}\left(x^{2}\right)}{\partial t^{2}}, & \Sigma x^{6}=\frac{3}{5} \Sigma x^{2}+_{I^{\frac{1}{2} \sigma}} \Sigma \frac{\partial^{4}\left(x^{2}\right)}{\partial t^{4}}, \text { u. s. w. }\end{cases}
$$

welche nach der Formel

$$
\Sigma x^{\mu}=\frac{\mu-3}{\mu-1} \Sigma x^{\mu-4}-\frac{\partial^{2} \Sigma x^{\mu-2}}{(\mu-1)(\mu-2) \partial t^{2}}
$$

aus einander hervorgehen. - Setzt man nun endlich in diesen letzteren (H.) nach (5.) für

$\Sigma x, \quad \Sigma \frac{\partial^{2} x}{\partial t^{2}}, \quad \Sigma \frac{\partial^{4} x}{\partial t^{4}}, \quad$ u. s.w. resp. die Werthe

$m \cdot y, \quad m \cdot \frac{\partial^{2} y}{\partial t^{2}}, \quad m \cdot \frac{\partial^{4} y}{\partial t^{4}}, \quad$ u.s. w., und für

$\Sigma x^{2}, \quad \Sigma \frac{\partial^{2}\left(x^{2}\right)}{\partial t^{2}}, \quad \Sigma \frac{\partial^{4}\left(x^{2}\right)}{\partial t^{4}}$, u. s.w., resp. die Werthe

$m^{2} y, \quad m^{2} \frac{\partial^{2}\left(y^{2}\right)}{\partial t^{2}}, m^{2} \cdot \frac{\partial^{4}\left(y^{2}\right)}{\partial t^{4}}$, u. s. w., so kommt: 


$$
\left\{\begin{aligned}
& \Sigma x^{3}=\boldsymbol{S}_{3}=-\frac{1}{2} m \frac{\partial^{2} y}{\partial t^{2}}, \boldsymbol{S}_{5}=\frac{1}{2} m y+\frac{m}{24} \frac{\partial^{4} y}{\partial t^{4}}, \text { u. s. w. } \\
& \boldsymbol{S}_{4}=\frac{1}{3} p-\frac{m^{2}}{6} \frac{\partial^{2}\left(y^{2}\right)}{\partial t^{2}}, \quad \boldsymbol{S}_{6}=\frac{3 m^{2}}{5} y^{2}+\frac{m^{2}}{120} \frac{\partial^{4}\left(y^{2}\right)}{\partial t^{4}}, \text { u. s. w. *) }
\end{aligned}\right.
$$

und wenn man hier die Differenzialquotienten von $y$ resp. $y^{2}$ nach $\left(F_{0}\right)$ in die ungeraden, resp. geraden Potenzen von $\boldsymbol{y}$ umsetzt, so erhält man $\boldsymbol{S}_{3}, \boldsymbol{S}_{5}$, $\boldsymbol{S}_{7}$, u. s. w. in ungerade, $\boldsymbol{S}_{4}, \boldsymbol{S}_{6}, \boldsymbol{S}_{8}$, u.s. w. in gerade ganze Functionen von $y$ ausgedrückt, z. B.

$$
\left.S_{3}=m^{3} y^{3}, \quad S_{4}=\frac{1}{3}\left(p-m^{4}\right)+m^{4}\right)^{4}, \text { u. s. w. }
$$

Nachdem wir so die Natur der Potenzsummen $\boldsymbol{S}_{\boldsymbol{\mu}}$ erkannt haben, nämlich, dafs sie ganze Functionen von $y$ sind, die auf den $\mu$ ten Grad steigen, und nur ungerade oder nur gerade Potenzen von $y$ enthalten, je nachdem $\mu$ ungerade oder gerade ist, wollen wir eine Recursionsformel aufstellen, nach der man unmittelbar jede Potenzsumme aus den frühern berechnen kann. Da $\boldsymbol{S}_{\mu}$ eine ganze Function von $\boldsymbol{y}$ vom $\mu$ ten Grade ist, so erhält man durch zweimalige Differenziation nach $t$

$$
\frac{\partial^{2} S_{\mu}}{\partial t^{2}}=\frac{\partial^{2} S_{\mu}}{\partial y^{2}}\left(\frac{\partial y}{\partial t}\right)^{2}+\frac{\partial S_{\mu}}{\partial y} \cdot \frac{\partial^{2} y}{\partial t^{2}}=m^{2}\left(1-y^{4}\right) \cdot \frac{\partial^{2} S_{\mu}}{\partial y^{2}}-2 m^{2} y^{3} \cdot \frac{\partial S_{\mu}}{\partial y},
$$

wo $\frac{\partial S_{\mu}}{\partial y}$ und $\frac{\partial^{2} S_{\mu}}{\partial y^{2}}$ ebenfalls ganze Functionen von $y$ vom $\mu-1$ ten resp. $\mu-2$ ten Grade sind. Von der andern Seite ist auch $\frac{\partial^{2} S_{\mu}}{\partial t^{2}}=\Sigma \frac{\partial^{2}\left(x^{\mu}\right)}{\partial t^{2}}$ aber

$$
\begin{aligned}
\frac{\partial^{2}\left(x^{\mu}\right)}{\partial t^{2}} & =\mu(\mu-1) x^{\mu-2}\left(\frac{\partial x}{\partial t}\right)^{2}+\mu x^{\mu-1} \cdot \frac{\partial^{2} x}{\partial t^{2}} \\
& =\mu(\mu-1) x^{\mu-2}\left(1-x^{4}\right)-2 \mu x^{\mu+2} \\
& =\mu(\mu-1) x^{\mu-2}-\mu(\mu+1) x^{\mu+2}, \quad \text { also } \\
\frac{\partial^{2} S_{\mu}}{\partial t^{2}} & =\mu(\mu-1) S_{\mu-2}-\mu(\mu+1) S_{\mu+2} ;
\end{aligned}
$$

dieser Werth von $\frac{\partial^{2} S_{\mu}}{\partial t^{2}}$ mit dem vorhin erhaltenen verglichen, liefert

$$
m^{2}\left(1-y^{4}\right) \cdot \frac{\partial^{2} S_{\mu}}{\partial y^{2}}-2 m^{2} y^{3} \cdot \frac{\partial S_{\mu}}{\partial y}=\mu(\mu-1) S_{\mu-2}-\mu(\mu+1) S_{\mu+2}
$$

hieraus folgt, wenn man noch $\mu-2$ statt $\mu$ setzt:

*) Die rechten Seiten dieser Gleichungen (I.) sind genau ebenso formirt, wie die der Gleichungen $(\boldsymbol{H}$.) und gehen unmiltelbar aus letzteren hervor, wenn man die Summenzeichen $\Sigma$ rechts fortläfst und statt $x$ überall rechts $m y$ stalt $x^{2}, m^{2} y^{2}$ schreibt. 
(6.)

$$
\boldsymbol{S}_{\mu}=\frac{\mu-3}{\mu-1} \boldsymbol{S}_{\mu-4}+\frac{m^{2}}{(\mu-1)(\mu-2)}\left\{2 y^{3} \frac{\partial S_{\mu-2}}{\partial y}-\left(1-y^{4}\right) \frac{\partial^{2} S_{\mu-2}}{\partial y^{2}}\right\} .
$$

Dies ist die verlangte Recursionsformel, nach welcher man aus $\boldsymbol{S}_{\mu-4}$ und $\boldsymbol{S}_{\mu-2}$ sogleich $\boldsymbol{S}_{\mu}$ ableiten kann. Da schon $\boldsymbol{S}_{0}=\boldsymbol{p}, \boldsymbol{S}_{1}=m \boldsymbol{y}, \boldsymbol{S}_{2}=\boldsymbol{m}^{2} \boldsymbol{y}^{2}, \boldsymbol{S}_{3}=\boldsymbol{m}^{3} \mathbf{y}^{3}$ gefunden worden ist, so leitet man hiernach die folgenden Potenzsummen ab:

$$
\begin{aligned}
& \boldsymbol{S}_{4}=\frac{1}{3} \boldsymbol{S}_{1}+\frac{m^{2}}{2 \cdot 3}\left(2 y^{3} \frac{\partial S_{2}}{\partial y}-\left(1-y^{4}\right) \frac{\partial^{2} S_{2}}{\partial y^{2}}\right) \\
&=\frac{1}{3} p+\frac{m^{2}}{2 \cdot 3}\left(4 m^{2} y^{4}-2 m^{2}\left(1-y^{4}\right)\right)=\frac{1}{3}\left(p-m^{4}\right)+m^{4} y^{4} \\
& \boldsymbol{S}_{5}=\frac{1}{2}\left(m-m^{5}\right) y+m^{5} y^{5} \\
& \boldsymbol{S}_{6}=\frac{3 m^{2}-3 m^{6}}{5} y^{2}+m^{6} y^{6}, \text { u. s. w. z. B. noch } \\
& \boldsymbol{S}_{8}=\frac{25 p-28 m^{4}+3 m^{8}}{3 \cdot 5 \cdot 7}+\frac{4}{5}\left(m^{4}-m^{8}\right) y^{4}+m^{8} y^{8} \\
& \boldsymbol{S}_{10}=\frac{7 m^{2}-9 m^{6}+2 m^{10}}{15} y^{2}+\frac{5\left(m^{6}-m^{10}\right)}{5} y^{6}+m^{10} y^{11} \\
& \text { u. s. w. f. }
\end{aligned}
$$

Betrachtet man das Bildungsgeselz dieser Ausdrücke, so wird man leicht die Richtigkeit der folgenden Bemerkungen einsehen. Die Coëfficienten der verschiedenen Potenzen von $\boldsymbol{y}$ in $\boldsymbol{S}_{\mu}$ sind ganze Functionén von $\boldsymbol{m}$, so dafs sich $\boldsymbol{S}_{\mu}$ als eine ganze Function der beiden Gräfsen $\boldsymbol{n}$ und $\boldsymbol{y}$ betrachten läfst, und zwar kommen nur diejenigen Potenzen sowohl von $m$ als von $y$ vor, deren Exponenten $\equiv \mu(\bmod .4)$; die Norm $p$ erscheint nur, wenn $\mu$ durch 4 theilbar ist, und zwar kommt sie dann nur linear und weder in $m$ noch in $y$ mulliplicirt vor; der Coefficient von $\boldsymbol{p}$ in $\boldsymbol{S}_{4 \mu}$ ist

$$
=\frac{1 \cdot 5 \cdot 9 \cdot 13 \cdots(4 \mu-3)}{3 \cdot 7 \cdot 11 \cdot 15 \cdots(4 \mu-1)}
$$

und der von $m^{2} y^{2}$ in $\boldsymbol{S}_{4 \mu-2}$ ist

$$
=\frac{3 \cdot 7 \cdot 11 \cdots(4 \mu-5)}{5 \cdot 9 \cdot 13 \cdots(4 \mu-3)}
$$

der Coëfficient von $y^{\mu}$ in $S_{\mu}$ ist $=m^{\mu}$, der von $y^{\mu-4}, \frac{\mu m^{\mu-4}\left(1-m^{4}\right)}{10}$, der von $y^{\mu-8}$ ebenfalls in $\boldsymbol{S}_{\mu}$,

$$
=\frac{\mu m^{\mu-8}\left(1-m^{4}\right)\left[3 \mu-2-(3 \mu-22) m^{4}\right]}{24 \cdot 25}, \text { u. s.w. }
$$

Man kann, wie schon oben bemerkt, die Potenzsummen $\boldsymbol{S}_{\mu}$ statt nach Potenzen von $y$ auch nach den Differenzialquotienten von $y$ resp. $y^{2}$ ordnen. 
Bei dieser zweiten Darsteliung, welche die einfachere ist, geschieht die successive Bildung nach der Formel

$$
\boldsymbol{S}_{\mu}=\frac{\mu-3}{\mu-1} \boldsymbol{S}_{\mu-4}-\frac{m^{2}}{(\mu-1)(\mu-2)} \frac{\partial^{2} S_{\mu-2}}{\partial u^{2}}
$$

wo $\partial u=m \partial \ell$ gesetzt worden ist, so dafs also die Differenzialquotienten von $y$ nach $u$ aus der Gleichung $\frac{\partial y}{\partial u}=\sqrt{ }\left(1-y^{4}\right)$ abzuleiten sind: dieselben ergeben sich demnach, was zu bemerken ist, aus den Differenzialquotienten von $\boldsymbol{x}$ nach $\boldsymbol{t}$, wenn man in diesen letzteren nach geschehener Differenziation $\boldsymbol{y}$ an die Stelle von $x$ setzt, also aus (E.), wenn man dort rechts $y$ statt $x$ schreibt. Man erhält hiernach

$$
\begin{aligned}
& \boldsymbol{S}_{4}=\frac{1}{3} \boldsymbol{p}-\frac{1}{6} m^{4} \frac{\partial^{2}\left(x^{2}\right)}{\partial t^{2}}, \quad \boldsymbol{S}_{5}=\frac{1}{2} m x+\frac{1}{24} m^{5} \frac{\partial^{4} x}{\partial t^{4}} \\
& \boldsymbol{S}_{6}=\frac{3}{5} \boldsymbol{m}^{2} x^{2}+_{T^{\frac{1}{2}} m^{6}} \frac{\partial^{4}\left(x^{2}\right)}{\partial t^{4}}, \text { u. s. w., }
\end{aligned}
$$

wo nach geschehener Differenziation überall $y$ statt $x$ zu schreiben ist. Da die numerischen Coëfficienten in diesen Ausdrücken genau dieselben sind, wie in $(\boldsymbol{G}$.), so erhalten wir hieraus folgenden allgemeinen Satz:

„Die Summe der $\mu$ ten Potenzen $\boldsymbol{S}_{\mu}$, der Wurzeln der Gleichung $y=\frac{\boldsymbol{U}}{\boldsymbol{V}}$ ergiebt sich, wenn man $x^{\mu}$ in die Differenzialquotienten von $x$ oder $x^{2}$ nach $t$ ausdrückt ${ }^{*}$ ), sodann die constanten Glieder mit $p$, die kten Differenzialquotienten von $x$ mit $m^{k+1}$ und die kten Differenzialquotienten von $x^{2}$ mit $m^{k+2}$ multiplicirt, endlich nach geschehener Differenziation $y$ an die Stelle von $x$ setzt."

Das Gesetz der Coëfficienten in der Entwickelung von $\boldsymbol{x}^{\mu}$ ist von $\mathbf{J} a c o b i$ (Fundamenta nova pag. 126 seq.) angegeben worden; er zeigt, dafs dieselben aus der Entwickelung der Potenzen von $\boldsymbol{t}$ nach Potenzen von $\boldsymbol{x}$ hervorgehen, also in unserem Falle aus der Entwickelung der Potenzen von $\int_{0}^{x} \frac{\partial x}{\sqrt{\left(1-x^{4}\right)}}$. Bei dieser Gelegenheit will ich einen Druckfehler in den betreffenden Formeln zur Verbesserung anzeigen: in „Fundamenta nova etc." auf Seite 126 ist statt der Constante in der 15ten Zeile zu lesen:

$$
-\left(\boldsymbol{R}_{n-2}^{(4)} \frac{U^{(2)}}{\Pi 4}+\boldsymbol{R}_{n-3}^{(6)} \frac{U^{(4)}}{\Pi 6}+\ldots+\frac{U^{(2 n-2)}}{\Pi 2 n}\right),
$$

*) wo $x$ durch die Gleichung $\frac{\partial x}{\partial t}=\sqrt{ }\left(1-x^{4}\right)$ gegeben ist. 
14. Eisenstein, zur. Theoric der Lemniscatenfunct. und Transformation. 201

oder was nach der dortigen Bezeichnung dasselbe ist:

$$
-\left(\boldsymbol{R}_{n-2}^{(4)} \cdot \frac{1}{3 \cdot 4}+\boldsymbol{R}_{n-3}^{(6)} \frac{S_{1}^{(2)}}{5 \cdot 6}+\ldots+\frac{S_{n-2}^{(2)}}{(2 n-1) 2 n}\right)
$$

dort sind die Factoren $\boldsymbol{R}_{n-2}^{(4)}, \boldsymbol{R}_{n-3}^{(6)}$, u. s. w. fortgelassen. Diese Constante reducirt sich übrigens für die Lemniscate auf ein einziges Glied und wird, wie schon bemerkt, $=0$ oder $=\frac{1 \cdot 5 \cdot 9 \cdots(4 \mu-3)}{3 \cdot 7 \cdot 11 \cdots(4 \mu-1)}$. Um die erwähnten Formeln auf die schnellste Weise zu erhalten, setze man in die allgemeine Gleichung

$$
(\alpha .) \quad \frac{\partial^{2} \psi}{\partial t^{2}}=\left(1-x^{4}\right) \frac{\partial^{2} \psi}{\partial x^{2}}-2 x^{3} \frac{\partial \psi}{\partial x}
$$

zuerst $\psi=\frac{t^{n}}{n !} ;$ man erhält dann:

$$
\frac{t^{n-2}}{(n-2) !}=\left(1-x^{4}\right) \frac{\partial^{2}\left(\frac{t^{n}}{n !}\right)}{\partial x^{2}}-2 x^{3} \frac{\partial\left(\frac{t^{n}}{n !}\right)}{\partial x} .
$$

Diese Gleichung werde $\mu$ mal auf beiden Seiten nach $x$ differenziirt, und nach geschehener Differenziation $x=0$ gesetzt, wobei zu bemerken, dafs die Differenzialquotienten von $1-x^{4}$ mit Ausnahme des Oten und 4ten und die von $x^{3}$ mit Ausnahme des dritten verschwinden. Man erhält:

$$
\frac{\partial^{\mu}\left(\frac{t^{n-2}}{(n-2) !}\right)}{\mu ! \partial x^{\mu}}=\frac{\partial^{\mu+2}\left(\frac{t^{n}}{n !}\right)}{\mu ! \partial x^{\mu+2}}-\frac{\partial^{\mu-2}\left(\frac{t^{n}}{n !}\right)}{(\mu-4) ! \partial x^{\mu-2}}-\frac{2 \partial^{\mu-2}\left(\frac{t^{n}}{n !}\right)}{(\mu-3) ! \partial x^{\mu-2}}
$$

oder :

$$
\text { (ß.) } \frac{\partial^{\mu}\left(\frac{t^{n-2}}{(n-2) !}\right)}{(\mu-1) ! \partial x^{\mu}}=\mu(\mu+1) \frac{\partial^{\mu+2}\left(\frac{t^{n}}{n !}\right)}{(\mu+1) ! \partial x^{\mu+2}}-\mu(\mu-1) \frac{\partial^{\mu-2}\left(\frac{t^{n}}{n !}\right)}{(\mu-3) ! \partial x^{\mu-2}},
$$

für $x=0$. Sodann nehme man in obiger Formel $\psi=x^{\mu}$, so dafs

$$
\text { (r.) } \frac{\partial^{2}\left(x^{\mu}\right)}{\partial t^{2}}=-\mu(\mu+1) x^{\mu+2}+\mu(\mu-1) x^{\mu-2} \text {. }
$$

Setzt man nun endlich

$$
\begin{aligned}
& x^{\mu}=(-1)^{\frac{1}{2}(\mu-1)} \sum_{n=0} \boldsymbol{P}_{n}^{(\mu)} \frac{\partial^{n} x}{\partial t^{n}}, \quad \text { oder } \\
& x^{\mu}=(-1)^{\frac{1}{(}(\mu-2)} \sum_{n=0} \boldsymbol{P}_{n}^{(\mu)} \frac{\partial^{n}\left(x^{2}\right)}{\partial t^{n}}+\text { Const. }
\end{aligned}
$$

je nachdem $\mu$ ungerade oder gerade ist, so erhält man nach $(\gamma$.)

$$
\begin{gathered}
(-1)^{\frac{1}{2}(\mu-1)} \boldsymbol{P}_{n-2}^{(\mu)}=-\mu(\mu+1)(-1)^{\frac{1}{d}(\mu+1)} \boldsymbol{P}_{n}^{(\mu+2)}+\mu(\mu-1)(-1)^{\frac{1}{2}(\mu-3)} \boldsymbol{P}_{n}^{(\mu-2)} \\
\text { Crelle's Journal f. d. M. Bd. XXX. Heft 3. } \\
26
\end{gathered}
$$$$
26
$$ 
202 14. Eisenstein, zur Theorie der Lemniscatenfunct. und Transformation.

resp.

$$
(-1)^{\frac{1}{2}(\mu-2)} \boldsymbol{P}_{n-2}^{(\mu)}=-\mu(\mu+1)(-1)^{\frac{1}{2} u} \boldsymbol{P}_{n}^{(\mu+2)}+\mu(\mu-1)(-1)^{\frac{1}{1}(\mu-4)} \boldsymbol{P}_{n}^{(\mu-2)},
$$

also in beiden Fällen:

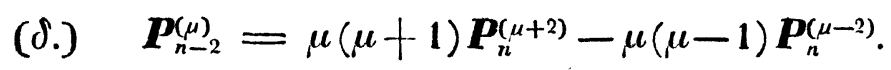

Dieser Recursionsgleichung genügt nach $(\beta$.

$$
\text { (E.) } \left.\quad \boldsymbol{P}_{n}^{(\mu)}=\frac{\partial^{\mu}\left(\frac{t^{n+1}}{(n+1) !}\right)}{(\mu-1) ! \partial x^{\mu}}, \quad \text { oder }=\frac{\partial^{\mu}\left(\frac{t^{n+2}}{(n+2) !}\right)}{(\mu-1) ! \partial x^{\mu}} \text { (für } x=0\right),
$$

und da dieser Werth slimmt, wenn man $\mu=1, \mu=2,3$ und 4 setzt, während $n$ allgemein bleibt ${ }^{*}$ ), so gilt er auch für alle Werthe von " und $n$, und man erhält:

$$
x^{\mu}=(-1)^{\frac{1}{1}(\mu-1)} \sum_{n=0} \frac{1}{(\mu-1) !(n+1) !} \frac{\partial^{n} x}{\partial t^{n}}\left(\frac{\partial^{\mu}\left(t^{n+1}\right)}{\partial x^{\mu}}\right)_{x=0}
$$

oder

$$
=(-1)^{\frac{1}{(2}(\mu-2)} \sum_{n=0} \frac{1}{(\mu-1) !(n+2) !} \frac{\partial^{n}\left(x^{2}\right)}{\partial t^{n}}\left(\frac{\partial^{\mu}\left(t^{n+2}\right)}{\partial x^{\mu}}\right)_{x=0}+C,
$$

je nachdem $\mu$ ungerade oder gerade ist; übrigens ist $\boldsymbol{C}=0$ oder $\boldsymbol{C}=$ $\frac{1 \cdot 5 \cdot 9 \cdots(\mu-3)}{3 \cdot 7 \cdot 11 \cdots(\mu-1)}$, je nachdem $\mu \equiv 2$ oder $\equiv 0$ (mod. 4) ist. Zu bemerken ist, dafs in diesen Formeln (8.) diejenigen Coëfficienten von selbst verschwinden, für welche nicht $n+1$ resp. $n+2 \equiv \mu$ (mod. 4) ist.

Für die $\mu$ ten Potenzsummen ergiebt sich nun hieraus durch Anwendung des oben aufgestellten allgemeinen Satzes:

$$
\mathbf{S}_{\mu}=\Sigma \boldsymbol{x}^{\mu}=(-1)^{\mathbf{z}(\mu-\sigma)} \sum_{n=0} \frac{m^{n+\sigma}}{(\mu-1) !(n+\sigma) !} \frac{\partial^{\mu}\left(\boldsymbol{t}^{n+\sigma}\right)}{\partial \boldsymbol{x}^{\mu}} \frac{\partial^{n}\left(y^{\sigma}\right)}{\partial \boldsymbol{u}^{n}}+\boldsymbol{C}_{\boldsymbol{p}},
$$

wo $\sigma=1$ oder $=2$, je nachdem $\mu$ ungerade oder gerade ist, $\frac{\partial y}{\partial u}=\sqrt{ }\left(1-y^{*}\right)$, $C=0$ für $\mu \equiv 1,2,3$ (mod.4), dagegen $C=\frac{1 \cdot 5 \cdot 9 \cdots(\mu-3)}{3 \cdot 7 \cdot 11 \cdots(\mu-1)}$ für $\mu \equiv 0$ (mod. 4), und wo nach geschehener Differenziation rechts $x=0$ gesetzt werden mufs. - Man kann auch noch die Summen der negativen Potenzen $\Sigma x^{-\mu}$ erhalten, wenn man $\frac{1}{y}$ statt $y$ schreibt, denn da $y$ in $\frac{1}{y}$ übergeht, wenn $\frac{1}{x}$

*) denn für $\mu=1, \mu=2$ erhält man nach den Werthen von $n$ auf beiden Seilen der Formel 1, 0,0 , 0 , etc.; für $\mu=3$ kommt $0,0, \frac{1}{2}, 0,0, \ldots$, und für $\mu=4$ : $0,0, \frac{b}{b}, 0,0, \ldots$. 
statt $x$ geselzt wird, so erhält man $\Sigma x^{-\mu}$ aus dem Werthe von $\Sigma x^{\mu}$, wenn man in letzterem $\frac{1}{y}$ statt $y$ setzt. - Aus den allgemeinen Potenzsummen $\boldsymbol{S}_{\mu}$, welche noch das variable $y$ enthalten, ergeben sich speciell die Potenzsummen des. Zählers der Multiplicationsformel $\boldsymbol{U}=0$, welche wir durch $\boldsymbol{S}_{\boldsymbol{\mu}}^{\prime}$ bezeichnen, wenn man $y=0$ setzt; wird aber $y=0$ gesetzt, so kann man in (9.) statt $\frac{\partial^{n}\left(y^{\sigma}\right)}{\partial \iota^{n}}$ auch $\frac{\partial^{n}\left(x^{\sigma}\right)}{\partial t^{n}}$ schreiben, wenn nur dann nach geschehener Differenziation $x=0$ gesetzt wird. $S_{\mu}^{\prime}$ verschwindet immer, wenn $\mu \equiv 1,2$, oder $3(\bmod .4)$ ist; dagegen für die durch 4 theilbaren Werthe von $\mu$ erhält man:

$$
\begin{aligned}
\boldsymbol{S}_{\mu}^{\prime}= & -\Sigma \frac{m^{n+2}}{(\mu-1) !(n+2) !} \frac{\partial^{\mu}\left(t^{n+2}\right)}{\partial x^{\mu}} \frac{\partial^{n}\left(x^{2}\right)}{\partial t^{n}} \quad\{x=t=0\} \\
& +\frac{1 \cdot 5 \cdot 9 \ldots(\mu-3)}{3 \cdot 7 \cdot 11 \ldots(\mu-1)} p .
\end{aligned}
$$

Es verschwinden in der Summe alle diejenigen Glieder, für welche nicht $n \equiv$ ? (mod. 4) ist, so dafs nur diejenigen Potenzen von $m$ bleiben, deren Exponenten durch 4 theilbar sind; man kann also auch setzen:

$$
\left(10^{\prime} .\right) \quad \boldsymbol{S}_{4 \mu}^{\prime}=-\sum_{n=1}^{n=\mu} \frac{m^{4 n}}{(4 \mu-1) !(4 n) !} \frac{\partial^{4 \mu}\left(t^{4 n}\right)}{\partial x^{4 \mu}} \frac{\partial^{4 n-2}\left(x^{2}\right)}{\partial t^{4 n-2}}+\frac{1 \cdot 5 \cdots(4 \mu-3)}{3 \cdot 7 \cdots(4 \mu-1)} p .
$$

Es ist zugleich $4 \mu$ statt $\mu$ geschrieben worden, weil es sich nur um die durch 4 theilbaren Werthe von $\mu$ handelt. Diese Formeln (10.) oder (10'.) geben die von Null verschiedenen Potenzsummen der Wurzeln der Gleichung

$$
x^{p-1}+B_{1} x^{p-5}+B_{2} x^{p-9}+\ldots+A_{1} x^{4}+m=0,
$$

und wenn man $\boldsymbol{S}_{4 \mu}^{\prime}=4 \cdot \boldsymbol{T}_{\mu}$ setzt, so stellt offenbar $\boldsymbol{T}_{\mu}$ die Summe der $\mu$ ten Potenzen der folgenden Gleichung dar:

$$
z^{\frac{1}{4}(p-1)}+B_{1} z^{\frac{1}{2}(p-1)-1}+\ldots+A_{1} z+m=0,
$$

welche aus der obigen durch die Substitution $x^{4}=z$ hervorgeht; diese Potenzsummen der Wurzeln der Gleichung in $z$ sind übrigens

$$
\boldsymbol{T}_{\mu}=\frac{1}{4} \boldsymbol{S}_{4 \mu}^{\prime}=\Sigma \varphi\left(\frac{r \omega}{m}\right)^{4 \mu}
$$

wo $\boldsymbol{r}$ in der Summe ein Viertelrestensystem (mod. $m$ ) repräsentirt; hiernach findet man: 
204 14. Eisenstein, zur Theorie der Lemniscatenfunct. und Transformution.

$$
\begin{aligned}
& \Sigma \varphi\left(\frac{r \omega}{m}\right)^{4}=T_{1}=\frac{p-m^{4}}{12} \\
& \Sigma \varphi\left(\frac{r \omega}{m}\right)^{8}=T_{2}=\frac{1}{4} \cdot \frac{1 \cdot 5}{3 \cdot 7} p-1_{15}^{1} m^{4}+\frac{1}{4} \cdot \frac{1}{35} m^{8} \\
& \Sigma \varphi\left(\frac{r \omega}{m}\right)^{12}=T_{3}=\frac{1}{4} \cdot \frac{1 \cdot 5 \cdot 9}{3 \cdot 7 \cdot 11} p-\frac{11 m^{4}}{3 \cdot 4 \cdot 25}+\frac{3 m^{8}}{2 \cdot 7 \cdot 25}-\frac{m^{12}}{2 \cdot 3 \cdot 11 \cdot 25}
\end{aligned}
$$

u. s. w.

Aus den Potenzsummen $\boldsymbol{T}_{1}, \boldsymbol{T}_{2}$, u. s. w. kann man nun die Coëfficienten $\boldsymbol{B}_{1}$, $\boldsymbol{B}_{2}$, u. s. w. berechnen, und zwar nach den von Newton gegebenen Formeln

$$
\begin{gathered}
B_{1}=-T_{1}, \quad 2 B_{2}=-T_{2}-B_{1} T_{1}, \quad 3 B_{3}=-T_{3}-B_{1} T_{2}-B_{2} T_{1}, \\
4 B_{4}=-T_{4}-B_{1} T_{3}-B_{2} T_{2}-B_{3} T_{1}, \text { u. s. w. }
\end{gathered}
$$

Hiernach findet man z. B.

$$
\begin{aligned}
\boldsymbol{B}_{1} & =\frac{-p+m^{4}}{12} \\
\boldsymbol{B}_{2} & =\frac{p^{2}}{2 \cdot 144}-\frac{5}{14 \cdot 12} p+m^{4}\left(\frac{-p}{144}+\frac{1}{30}\right)-\frac{m^{8}}{8 \cdot 35 \cdot 36} \\
& =\frac{1}{56 \cdot 180}\left(-m^{8}-70 m^{4} p+35 p^{2}+336 m^{4}-300 p\right), \text { u. s. w. }
\end{aligned}
$$

Auf eine ähnliche Weise findet man die Coëfficienten $\boldsymbol{A}_{1}, \boldsymbol{A}_{2}$, u. s. w. :

$$
\begin{aligned}
& \boldsymbol{A}_{1}=\frac{m}{60}\left(-m^{4}-5 p+6\right) \\
& \boldsymbol{A}_{2}=\frac{m}{56 \cdot 180}\left(-m^{8}+14 m^{4} p+35 p^{2}-84 m^{4}-384 p+420\right), \text { u. s. w. }
\end{aligned}
$$

Setzt man, was erlaubt ist,

$$
\boldsymbol{B}_{\mu}=\alpha_{\mu}+\beta_{\mu} m^{4}+\gamma_{\mu} m^{8}+\ldots+\lambda_{\mu} m^{4 \mu},
$$

so erhält man aus der Verbindung von $\left(10^{\prime}\right.$.) mit den Newtonschen Formeln z. B.

$$
\mu \alpha_{\mu}=-\frac{1}{4} \nu\left[\frac{1 \cdot 5 \cdots(4 \mu-3)}{3 \cdot 7 \cdots(4 \mu-1)}+\frac{1 \cdot 5 \cdots(4 \mu-7)}{3 \cdot 7 \cdots(4 \mu-5)} \alpha_{1}+\ldots+\frac{1 \cdot 5}{3 \cdot 7} \alpha_{\mu-2}+\frac{1}{3} \alpha_{\mu-1}\right] \text {, }
$$

u. s. w. für $\beta_{\mu}, \gamma_{\mu}$ etc. ähnliche Formeln.

Wir stellen noch die folgenden Bemerkungen über die Coëfficienten des Zählers und Nenners der Multiplicationsformeln für die Lemniscate zusammen:

a) Der allgemeine Coëfficient $\boldsymbol{B}_{\mu}$ des. Nennẹs ist als eine ganze Function der beiden Variabeln $\boldsymbol{m}^{4}$ und $\boldsymbol{p}$ von der $\mu$ ten Ordnung anzusehen, in welcher das constante Glied fehlt; der allgemeine Coëfficient $\boldsymbol{A}_{\mu}$ des Zählers ist gleich dem mfachen einer ganzen Function $\mu$ ter Ordnung der beiden Variabeln $m^{4}$ und $p$; die Coëficienten der Potenzen und Producte von $m^{4}$ und $p$ in diesen ganzen Functionen sind rein numerisch, reell, und hängen weder von $m$ noch von $p$ ab, ihr Gesetz bleibt noch unbekannt und kann auch auf 
einem Wege, wie der hier eingeschlagene, schwerlich gefunden werden; man könnte zwar die Werthe von $\boldsymbol{T}_{\mu}=\frac{1}{4} \boldsymbol{S}_{4 \mu}^{\prime}$ nach $\left(10^{\prime}\right.$.) in die independenten Ausdrücke der Coëfficienten durch die Potenzsummen

$$
\begin{aligned}
\boldsymbol{B}_{\mu}= & \sum \frac{(-1)^{\alpha+\beta+\gamma+\cdots} \boldsymbol{T}_{1}^{\alpha} \boldsymbol{T}_{2}^{\beta} \boldsymbol{T}_{3}^{\gamma} \cdots}{\mathbf{1}^{\alpha} \cdot 2^{\beta} \cdot 3^{\gamma} \cdots \alpha ! \beta ! \gamma ! \cdots \cdots} \\
& \{\text { conditione } \alpha+2 \beta+3 \gamma+\cdots \cdots=n\}
\end{aligned}
$$

einsetzen, aber ein so erhaltenes Gesetz der Coëfficienten würde ihre Natur mehr verhüllen als entdecken.

b) Obgleich in den Coëfficienten die Norm $p$ bis auf den $\mu$ ten Grad steigt, so enthalten die aus ihnen gebildeten Potenzsummen der Wurzeln doch $p$ nur linear und auch nicht in Potenzen von $m$ multiplicirt.

c) Die Coëfficienten brechen, vermöge ihrer Form, von selbst ab, sobald $\mu$ gröfser als $\frac{1}{4}(\boldsymbol{p}-1)$ ist. Nach dieser Bemerkung in Verbindung mit b) kann man unabhängig von den vorhergehenden Betrachtungen und von der Kenntnifs der Potenzsummen eine beliebige Anzahl Coëfficienten berechnen, indem man die in ihnen vorkommenden numerischen Coëfficienten als Unbekannte einführen und so viele Gleichungen bilden kann, als Unbekannte vorhanden sind. $\quad \boldsymbol{B}_{1}=\mathrm{T}_{\mathrm{T} 2}^{1}\left(m^{4}-p\right)$ und $A_{1}={ }_{6}^{\frac{1}{6}} m^{\prime}\left(-m^{4}-5 p+6\right)$ z. B. verschwinden für $m=1$, und werden $B_{1}=-1 \pm 2 i, A_{1}=1$ für $m=-1 \pm 2 i$, $p=5$; hieraus allein schon hätten diese beiden Coëfficienten gefunden werden können; denn setzte man $B_{1}=\alpha m^{4}+\beta p, A_{1}=m\left(\gamma m^{4}+\delta p+\varepsilon\right)$, so erhält man die fünf Gleichungen

$$
\begin{aligned}
\alpha+\beta=0, & (-1+2 i)^{4} \alpha+5 \beta=-1+2 i, \\
\gamma+\delta+\varepsilon=0, & (-1+2 i)^{5} \gamma+5(-1+2 i) \delta+(-1+2 i) \varepsilon=1, \\
& (-1-2 i)^{5} \gamma+5(-1-2 i) \delta+(-1-2 i) \varepsilon=1,
\end{aligned}
$$

wodurch die 5 Unbekannten $\alpha, \beta, \gamma, \delta, \varepsilon$ vollkommen bestimmt sind. Bei späteren Coëfficienten mufs man, um eine hinreichende Anzahl Gleichungen zu erhalten, wie schon bemerkt, noch die Eigenschaft b) hinzunehmen. Auch bedient man sich hierbei mit Vortheil der Beziehung zwischen den Coëfficienten des Zählers und Nenners (\$. 1.).

d) Wie oben bewiesen, sind für eine zweigliedrige Primzahl $m$ die Werthe von $\boldsymbol{A}_{\mu}$ und $\boldsymbol{B}_{\mu}$ durch $m$ theilbar.

e) In $B_{\mu}$ ist der Coëfficient von $p,=\frac{-1}{4 \mu} \frac{1 \cdot 5 \cdot 9 \cdots(4 \mu-3)}{3 \cdot 7 \cdot 11 \cdots(4 \mu-1)}$ der Coëfficient von $p^{\mu},=\frac{(-1)^{\mu}}{\mu ! 12^{\mu}}$, der von $p^{\mu-1},=\frac{5}{14} \frac{(-1)^{\mu-1}}{(\mu-2) ! 12^{\mu-1}}$. 
f) Wenn man in den Coëfficienten stalt $p$ eine beliebige Gröfse setzt, so bleibt die Multiplicationsformel immer noch richtig; Zähler und Nenner brechen aber nur dann ab, wenn $\boldsymbol{p}$ die Norm von $m$ ist.

Ich gebe diesen Versuch, so unvollkommen er auch sein mag, da bei der grofsen Schwierigkeit des Gegenstandes jeder Beitrag von Interesse sein kann.

Eine brauchbare Formel erhält man noch, wenn man die (9.) auf beiden Seiten nach $\boldsymbol{t}$ differenziirt:

$$
\Sigma\left(x^{\mu-1} \cdot \sqrt{ }\left(1-x^{4}\right)\right)=\frac{(-1)^{\frac{1}{2}(\mu-\sigma)}}{\mu !} \Sigma\left\{\frac{m^{n+\sigma+1}}{(n+\sigma) !}\left(\frac{\partial^{\mu}\left(t^{n+\sigma}\right)}{\partial x^{\mu}}\right)_{x=0)} \cdot \frac{\partial^{n+1}\left(y^{\sigma}\right)}{\partial \iota^{n+1}}\right\}
$$

5.

Die Methode des vorigen Paragraphen zur Bestimmung der symmetrischen Functionen der Wurzeln solcher Gleichungen, welche einer gegebenen Differenzialgleichung genügen, erstreckt sich viel weiter, als wir es hier gezeigt haben; sie läfst sich auf mehrere Probleme über elliptische Functionen und noch auf mannigfaltige andere Formen von Integralen algebraischer Functionen anwenden. Hier wollen wir nur noch in aller Kürze mit ihrer Hülfe die symmetrischen Verbindungen der Wurzeln derjenigen Gleichungen bestimmen, welche bei der Transformation elliptischer Functionen mit beliebigen Moduln vorkommen *).

I. Wenn $\frac{\partial x}{\partial t}=\sqrt{ }\left(\left(1-x^{2}\right)\left(1-k^{2} x^{2}\right)\right)$, also $\frac{\partial^{2} x}{\partial t^{2}}=-\left(1+k^{2}\right) x+2 k^{2} x^{3}$, so hat man die allgemeine Gleichung

$$
\text { (1.) } \frac{\partial^{2} \psi}{\partial t^{2}}=\frac{\partial^{2} \psi}{\partial x^{2}}\left(1-\left(1+k^{2}\right) x^{2}+k^{2} x^{4}\right)+\frac{\partial \psi}{\partial x}\left(-\left(1+k^{2}\right) x+2 k^{2} x^{3}\right) \text {, }
$$

wo $\psi$ irgend eine Function von $x$ oder von $\boldsymbol{t}$ ist. Setzt man hier zuerst $\psi=x^{\mu}$, sodann $\psi=t^{h}$, so erhält man:

$$
\begin{aligned}
\text { (2.) } \frac{\partial^{2}\left(x^{\mu}\right)}{\partial t^{2}} & =\mu(\mu+1) \alpha x^{\mu+2}-\mu^{2} \beta x^{\mu}+\mu(\mu-1) x^{\mu-2}, \\
\text { (3.) } h(h-1) t^{h-2} & =\frac{\partial^{2}\left(t^{h}\right)}{\partial x^{2}}\left(1-\beta x^{2}+\alpha x^{4}\right)+\frac{\partial\left(t^{h}\right)}{\partial x}\left(-\beta x+2 \alpha x^{3}\right),
\end{aligned}
$$

wo der Kürze halber $k^{2}=\alpha, 1+k^{2}=\beta$ gesetzt worden ist. Differenziirt man die letztere Gleichung (3.) $\mu$ mal nach $x$ und setzt nach geschehener Differenziation $x=0$, so kommt

*) Man vergleiche auch besonders die „Notices sur les fonct. ellipt." von Jacobi im 3ten und 4ten Bande des Crelle'schen Journals. 


$$
\begin{aligned}
\boldsymbol{h}(\boldsymbol{h}-1) \frac{\partial^{\mu}\left(t^{h-2}\right)}{\mu ! \partial x^{\mu}}=\frac{\partial^{\mu+2}\left(t^{h}\right)}{\mu ! \partial x^{\mu+2}} & -\beta \frac{\partial^{\mu}\left(t^{h}\right)}{(\mu-2) ! \partial x^{\mu}}+\alpha \frac{\partial^{\mu-2}\left(t^{h}\right)}{(\mu-4) ! \partial x^{\mu-2}} \\
& -\beta \frac{\partial^{\mu}\left(t^{h}\right)}{(\mu-1) ! \partial x^{\mu}}+2 \alpha \frac{\partial^{\mu-2}\left(t^{h}\right)}{(\mu-3) ! \partial x^{\mu-2}}
\end{aligned}
$$

oder, wenn man reducirt,

$$
\begin{gathered}
\text { (4.) } \frac{\partial^{\mu}\left(\frac{t^{h-2}}{(h-2) !}\right)}{(\mu-1) ! \partial x^{\mu}}= \\
\qquad(\mu+1) \frac{\partial^{\mu+2}\left(\frac{t^{h}}{h !}\right)}{(\mu+1) ! \partial x^{\mu+2}}-\mu^{2} \beta \frac{\partial^{\mu}\left(\frac{t^{h}}{h !}\right)}{(\mu-1) ! \partial x^{\mu}}+\mu(\mu-1) \alpha \frac{\partial^{\mu-2}\left(\frac{t^{h}}{h !}\right)}{(\mu-3) ! \partial x^{\mu-2}} \\
\text { wird also } \frac{\partial^{\mu}\left(\frac{t^{h}}{h !}\right)}{(\mu-1) ! \partial x^{\mu}}=R_{h}^{\mu)} \text { gesetzt für } x=0 \text {, so hat man }
\end{gathered}
$$

$$
\left(4^{\prime} .\right) \quad \boldsymbol{R}_{h-2}^{(\mu)}=\mu(\mu+1) \boldsymbol{R}_{h}^{(\mu+2)}-\mu^{2} \beta \boldsymbol{R}_{h}^{(\mu)}+\mu(\mu-1) \alpha \boldsymbol{R}_{h}^{(\mu-2)} .
$$

II. Vermöge (2.) kann die folgende Entwickelung angenommen werden :

$$
\alpha^{\frac{1}{2}(\mu-o)} x^{\mu}=\sum_{h=0} \boldsymbol{P}_{h}^{(\mu)} \frac{\partial^{h}\left(x^{\sigma}\right)}{\partial t^{h}}-\text { Const. },
$$

wo ' $\sigma=1$ oder $=2$, je nachdem $\mu$ ungerade oder gerade ist; z. B. für ,$=1$ ist $x=x$, also $\boldsymbol{P}_{h}^{(1)}=1$ für $h=0$ und $=0$ für $h>0$; für $\mu=2$ ist $x^{2}=x^{2}$, also $\boldsymbol{P}_{h}^{(2)}=1$ für $h=0$ und $=0$ für $h>0$; für $\mu=3$ ist $\alpha x^{3}=\frac{1}{2} \beta x+\frac{1}{2} \frac{\partial^{2} x}{\partial t^{2}}$, also nimmt $\boldsymbol{P}_{h}^{(3)}$ für die aufeinanderfolgenden Werthe von $h$ die Werthe an: $\frac{1}{2} \beta, 0, \frac{1}{2}, 0,0,0$, u. s. w.; für $\mu=4$ wird

$$
\alpha x^{4}=\frac{2}{3} \beta x^{2}+\frac{1}{6} \frac{\partial^{2}\left(x^{2}\right)}{\partial t^{2}}-\frac{1}{8},
$$

also sind die Werthe von $\boldsymbol{P}_{h}^{(4)}$ die folgenden: $\frac{2}{3} \beta, 0, \frac{1}{\gamma}, 0,0,0$, u. s. w. Setzt man die obige Entwickelung in die Gleichung (2.), so kommt

$$
\text { (5.) } \quad \boldsymbol{P}_{h-2}^{(\mu)}=\mu(\mu+1) \boldsymbol{P}_{h}^{(\mu+2)}-\mu^{2} \beta \boldsymbol{P}_{h}^{(\mu)}+\mu(\mu-1) \alpha \boldsymbol{P}_{h}^{(\mu-2)} \text {. }
$$

Nach (4'.) genügt dieser Relation:

$$
\text { (6.) } \quad \boldsymbol{P}_{h}^{(\mu)}=\boldsymbol{R}_{h+\sigma}^{(\mu)},
$$

und wenn diese Übereinstimmung für 4 Anfangswerthe von $\mu$, während $h$ allgemein bleibt, nachgewiesen werden kann, so mufs sie allgemein stattinden, weil $\boldsymbol{P}^{(\mu+2)}$ genau ebenso aus den $\boldsymbol{P}$ mit den oberen Indices $\mu$ und $\mu-2$ berechnet wird, wie $R^{(\mu+2)}$ aus den $R$ mit den oberen Indices $\mu$ und $\mu-2$. Diese Übereinstimmung findet in der That für $\mu=1,2,3,4$ Statt, denn $\boldsymbol{R}_{h+1}^{(1)}, \boldsymbol{R}_{h+2}^{(2)}$, $\boldsymbol{R}_{h+1}^{(3)}, \boldsymbol{R}_{h+2}^{(4)}$ nehmen für $h=0,1,2,3, \ldots$ in inf. die Werthe an resp.: 
208 14. Eisenst ein, zur. Theorie der Lomniscatenfunct. und Transformation.

$$
\begin{aligned}
& \boldsymbol{R}_{h+1}^{(1)}=1, \quad 0,0,0,0, \ldots \ldots, \\
& \boldsymbol{R}_{h+2}^{(2)}=1, \quad 0,0,0,0, \ldots, \\
& \boldsymbol{R}_{h+1}^{(3)}=\frac{1}{2} \beta, 0, \frac{1}{2}, 0,0, \ldots ., \\
& \boldsymbol{R}_{h+2}^{(4)}=\frac{2}{3} \beta, 0, \frac{1}{6}, 0,0, \ldots .,
\end{aligned}
$$

und diese Werthe stimmen genau mit den obigen Anfangswerthen von $\boldsymbol{P}_{h}^{(\mu)}$ für $\mu=1,2,3$ und 4; also findet (6.) allgemein Statt. Wir erhalten demnach:

$$
\text { (7.) } \quad k^{\mu-\sigma} x^{\mu}=\sum_{h=0} \boldsymbol{R}_{h+\sigma}^{\mu)} \frac{\partial^{h}\left(x^{\sigma}\right)}{\partial t^{h}}-\text { Const., }
$$

wo

$$
\boldsymbol{R}_{h+\sigma}^{(\mu)}=\frac{1}{(h+\sigma) !(\mu-1) !}\left(\frac{\partial^{\mu}\left(t^{h+\sigma}\right)}{\partial x^{\mu}}\right)_{x=0} .
$$

Die Constante wird aus dem besondern Falle $x=0, t=0$ bestimmt; man findet

$$
\text { Const. }=\sum_{h=0} \boldsymbol{R}_{h+\sigma}^{(\mu)} \cdot\left(\frac{\partial^{h}\left(x^{\sigma}\right)}{\partial t^{h}}\right)_{t=1)} .
$$

III. Für jede ungerade Zahl $\boldsymbol{n}$ lassen sich, wie durch Jacobi und Abel bekannt, mehrere Werthe von $\lambda$ und zugehörige von $\boldsymbol{N}$ angeben, so dafs

$$
\frac{\partial y}{\partial t}=\boldsymbol{N} \cdot V\left(\left(1-y^{2}\right)\left(1-\lambda^{2} y^{2}\right)\right)=N \cdot \frac{\partial y}{\partial u}, \quad \partial u=N \cdot \partial t
$$

wird, während $y$ eine gebrochene rationale Function von $x$ ist, die mit $x$ zugleich verschwindet, und deren Zähler und Nenner auf den $n$ ten resp. $n-1$ ten Grad steigen. Es sei

$$
y=\frac{U}{V}=\frac{k}{N \lambda} \frac{x^{n}+B x^{n-2}+B_{2} x^{n-4}+\ldots}{x^{n-1}+A_{1} x^{n-3}+A_{2} x^{n-5}+\ldots}
$$

dafs $y$ diese Form annehmen mufs, ergiebt sich unmittelbar durch Einsetzen in die Differenzialgleichung und ist von Jacobi an mehreren Orten bemerkt worden. Die Wurzeln der Gleichung

$$
\begin{gathered}
U-V y=0, \text { d. h. } \\
x^{n}-\frac{N \lambda}{k} y \cdot x^{n-1}+B x^{n-2}-\text { etc. }=0
\end{gathered}
$$

seien durch

und die Summe

$$
x_{1}, x_{2}, x_{3}, \ldots x_{n}
$$

$$
\boldsymbol{F}\left(x_{1}\right)+\boldsymbol{F}\left(x_{2}\right)+\boldsymbol{F}\left(x_{3}\right)+\ldots+\boldsymbol{F}\left(x_{n}\right) \operatorname{durch} \sum_{x} \boldsymbol{F}(x)
$$

bezeichnet. Die Summe der Gröfsen $x_{1}, x_{2}, x_{3}, \ldots x_{n}$ ist $=\frac{N \lambda}{k} y$; die - Summe ihrer Combinationen zu je zweien $=B$, also die Summe ihrer Quadrate 
$=\frac{N^{2} \lambda^{2}}{k^{2}} y^{2}-2 B$. Hieraus gehen zwei Gleichungen hervor, die sich in die einzige folgende zusammenziehen lassen:

$$
\text { (8.) } \sum_{x} x^{\sigma}=\frac{N^{\sigma} \lambda^{\sigma}}{k^{\sigma}} y^{\sigma}-c,
$$

wo $\sigma=1$ und $=2$ zu nehmen, während nach diesen beiden Fällen $c$ resp. $=0$ oder $=2 \boldsymbol{B}$ zu setzen ist. Die Gleichung (8.) differenziire man auf beiden Seiten $h$ mal nach $t$, wobei zu bemerken, dafs $\partial u^{h}=N^{h} \partial t^{h}$, so kommt:

$$
\text { (9.) } \quad \sum_{x} \frac{\partial^{h}\left(x^{\sigma}\right)}{\partial t^{h}}=\frac{N^{h+\sigma} \lambda^{\sigma}}{k^{\sigma}} \frac{\partial^{h}\left(y^{\sigma}\right)}{\partial u^{h}} \text {; }
$$

für $h=0$ tritt die Gleichung (8.) an die Stelle von (9.)

IV. Werden jetzt in (7.) für $x$ nach der Reihe die Werthe $x_{1}, x_{2}$, $x_{3}, \ldots x_{n}$ gesetzt, dieselben summirt, und endlich für

$$
\sum_{x} \frac{\partial^{h}\left(x^{\sigma}\right)}{\partial t^{h}}
$$

die Werthe aus (8.) und (9.) eingesetzt, so kommt:

(10.) $\quad k^{\mu-\sigma} \sum_{x} x^{\mu}=\sum_{h=0}^{h=\mu-\sigma} R_{h+\sigma}^{(\mu)} \frac{N^{h+\sigma} \lambda^{\sigma}}{k^{\sigma}} \frac{\partial^{h}\left(y^{\sigma}\right)}{\partial u^{h}}-n \sum_{h=0}^{h=\mu-\sigma} R_{h+\sigma}^{(\mu)}\left(\frac{\partial^{h}\left(x^{\sigma}\right)}{\partial t^{h}}\right)_{t=0}-\boldsymbol{c} \boldsymbol{R}_{\sigma}^{(\mu)}$; $\sigma$ ist, wie schon bemerkt, $=1$ oder $=2$, je nachdem $\mu$ ungerade oder gerade ist; die Bedeutung der Coëfficienten $\boldsymbol{R}_{h+\sigma}^{(\mu)}$ ist oben angegeben, und $x, y$ rechts werden in $t$ resp. $u$ bestimmt durch die Differenzialgleichungen:

$$
\frac{\partial x}{\partial t}=\sqrt{ }\left(\left(1-x^{2}\right)\left(1-k^{2} x^{2}\right)\right) \quad \text { und } \quad \frac{\partial y}{\partial u}=\sqrt{ }\left(\left(1-y^{2}\right)\left(1-\lambda^{2} y^{2}\right)\right) \text {. }
$$

Da man nach der. lelzteren die vorkommenden Differenzialquotienten $\frac{\partial^{h}\left(y^{\sigma}\right)}{\partial u^{\sigma}}$, in Potenzen von $y$ umsetzen kann, so giebt die Formel (10.) die $\mu$ ten Potenzsummen der Wurzeln der Gleichung $\boldsymbol{U}-\boldsymbol{V} \boldsymbol{y}=0$ vollständig in ganzen Functionen von $y$ ausgedrückt. Aus den Potenzsummen kann man alle anderen symmetrischen Verbindungen dieser Wurzeln ableiten; man kann also namentlich die Coëfficienten des Zählers und Nenners $\boldsymbol{U}$ und $\boldsymbol{V}$ der Transformationsformel sämmtlich finden, und zwar mit Hülfe der vier Gröfsen

$$
\boldsymbol{k}, \lambda, \boldsymbol{N} \text { und } \boldsymbol{B} \text {. }
$$

Dieses letztere schwierige Problem ist zuerst von Jacobi auf einem ganz anderen Wege gelöset worden (Band 4.).

Für den Fall der Multiplication ist $\boldsymbol{n}^{2}$ statt $n$ zu selzen; ferner ist $\boldsymbol{N}=\boldsymbol{n}$, $\lambda=k$ und $\boldsymbol{B}=0$, also werden dann die symmetrischen Functionen blofs in $\boldsymbol{n}$ und $\boldsymbol{k}$ ohne Hülfe anderer Gröfsen ausgedrückt.

Crelle's Journal f. d. M. Bd. XXX. Heft 3. 
Mit Hülfe des Satzes von Lagrange über die Umkehrung der Reihen kann man der Gleichung (10.) mehrere andere Formen geben.

V. Wenn $n$ eine Primzahl ist, so kann man in (9.) statt $\lambda$ seine $n+1$ Werthe und statt $\boldsymbol{N}$ die zugehörigen Werthe setzen. Summirt man dann links und rechts alle diese Werthe von $\lambda$ und $\boldsymbol{N}$, so bekommt man links die analoge Reihe für die Multiplication, die sich wiederum unmittelbar nach (9.) finden läfst, wenn man $\lambda=k, N=n$ selzt. Wird nun ferner $t=0$ ) und $\boldsymbol{u}=\mathbf{0}$ genommen, so hat man links blofse Functionen von $k$, während rechts symmetrische Verbindungen der Gröfsen $\lambda$ und $\boldsymbol{N}$ stehen. Man kann also auf diese Art unendlich viele symmetrische Verbindungen der transformirten Moduln und der zugehörigen Multiplicatoren in $k$ ausdrücken. Die Coëfficienten der Modulargleichung erhält man aber daraus nicht, weil es sehr schwierig ist, die Werthe der $\boldsymbol{N}$ von denen der $\lambda$ zu trennen.

Dagegen kann man aus den Newtonschen Formeln alle Coëfficienten eliminiren und eine beliebige Anzahl Gleichungen bilden, welche blofs Potenzsummen und keine Coëfficienten mehr enthalten. Gesetzt man habe $\nu$ solcher Gleichungen gebildet, wo $\nu>3$ genommen werden mufs. Man setze nun in diese $\nu$ Gleichungen die Werthe der Potenzsummen der Wurzeln der Gleichung $\boldsymbol{U}=\mathbf{0}$ ein, welche aus (10.) hervorgehen, wenn man dort rechts nach geschehener Differenziation $y=0$ setzt. Die $\nu$ Gleichungen enthalten dann aufser $k$ noch die drei Grö̉sen $\lambda, \boldsymbol{N}$ und $\boldsymbol{B}$. Eliminirt man zwischen ihnen $\boldsymbol{N}$ und $\boldsymbol{B}$, so ist der gröfste gemeinschaftliche Theiler der hieraus hervorgehenden $\nu-2$ Gleichungen die Modulargleichung; der gröfste gemeinschaftliche Theiler der aus der Elimination von $\lambda$ und $\boldsymbol{B}$ hervorgehenden $\nu-$ ? Gleichungen ist die Gleichung zwischen $N$ und $k$, und der gröfste gemeinschaftliche Theiler derjenigen, welche durch die Elimination von $\lambda$ und $\boldsymbol{N}$ entstehen, ist eine Gleichung zwischen $\boldsymbol{B}$ und $\boldsymbol{k}$.

Wir gestehen, dafs diese Methode, die Modulargleichung zu finden, allerdings noch einer bedeutenden Ausbildung bedarf, wenn sie nicht für ein allyemeines $\boldsymbol{n}$ illusorisch werden soll.

Im October 1845. 


\section{Neuer Beweis der Summationsformeln.}

$\mathbf{D}_{\text {ie Beweise der Summationsformeln für die elliptischen Functionen müssen }}$ immer heuristischer Art sein, indem bei Formeln, deren Richtigkeit man a posteriori durch unmittelbare Substitution zu prüfen vermag, von einer Verification nicht die Rede sein kann. Durch diese ihre heuristische Natur haben jene Beweise den Vorzug, dafs sie die Wissenschaft mit Methoden bereichern, welche auch über den augenblicklichen $Z_{w}$ eck hinaus von Nutzen sein können. Obgleich daher durch die längst von anderen Mathematikern gegebenen Beweise der hier in Rede stehende Gegenstand als vollständig erledigt betrachtet werden kann, so möchte doch die folgende neue Behandlung desselben, in Rücksicht auf die Methode, nicht als ganz überflüssig erscheinen.

Die Summationsformeln für die Sinus lassen sich auf folgende Weise ableiten. Wenn $\operatorname{man} x=\sin t$ als diejenige Function von $t$ definirt, welche mit $t$ zugleich verschwindet und der Differenzialgleichung $\frac{\partial x}{\partial t}=v\left(1-x^{2}\right)$ genügt, so hat man

$$
\frac{\partial^{2} x}{\partial t^{2}}=-x, \quad \frac{\partial^{3} x}{\partial t^{3}}=-\sqrt{ }\left(1-x^{2}\right), \quad \text { u. s. w., }
$$

so dafs die geraden Differenzialquotienten von $x$ nach $t,= \pm x$, die ungeraden $= \pm \gamma\left(1-x^{2}\right)$ sind. Da nun nach dem Taylorschen Satze, wenn $y=\sin u$ gesetzt wird, $\sin (t+u)$ einer Reihe von folgender Form gleich ist:

$$
\alpha y+\beta \frac{\partial y}{\partial u}+\gamma \frac{\partial^{2} y}{\partial u^{2}}+\delta \frac{\partial^{3} y}{\partial u^{3}}+\text { etc., }
$$

wo die Coëfficienten blofs von $t$ abhängen, so kann man hier alle Glieder mit geraden Differenzialquotienten von $y$ nach $\boldsymbol{u}$ in ein Glied von der Form $\boldsymbol{A} y$, und alle Glieder mit ungeraden Differenzialquotienten in ein Glied von der Form $\boldsymbol{B}_{V}\left(1-y^{2}\right)$ zusammenziehen, und man erhält:

$$
\sin (\boldsymbol{l}+\boldsymbol{u})=\boldsymbol{A} \boldsymbol{y}+\boldsymbol{B}_{1}\left(1-\boldsymbol{y}^{2}\right)
$$

wo $\boldsymbol{A}$ und $\boldsymbol{B}$ nur von $\boldsymbol{t}$ abhängen. Differenziirt man diese Gleichung auf beiden Seiten nach $u$, so kommt

$$
\frac{\partial \sin (t+u)}{\partial \boldsymbol{t}}=\boldsymbol{A}_{1}\left(1-y^{2}\right)-B y,
$$

und setzt man endlich, um $\boldsymbol{A}$ und $\boldsymbol{B}$ zu bestimmen, in diesen beiden Gleichungen $u=0$, wobei zu bemerken; dafs für $u=0: y=0, \sqrt{ }\left(1-y^{2}\right)=1$, 
$\sin (t+u)=\sin t=x, \frac{\partial \sin (t+u)}{\partial u}=\frac{\partial x}{\partial t}=\sqrt{ }\left(1-x^{2}\right)$ wird, so ergiebt sich $\boldsymbol{B}=\boldsymbol{x}$ und $\boldsymbol{A}=\sqrt{ }\left(1-x^{2}\right)$, also hat man schliefslich

$$
\sin (t+u)=x \sqrt{ }\left(1-y^{2}\right)+y \sqrt{ }\left(1-x^{2}\right),
$$

wenn $x=\sin t, y=\sin u$ ist.

Eine ähnliche Methode soll in den folgenden Zeilen auf die elliptischen Functionen angewandt werden.

Wir definiren die elliptische Function $x=\varphi(t)$, welche mit $t$ zugleich verschwindet, durch die Differenzialgleichnng

$$
\text { (1.) } \frac{\partial x}{\partial t}=\Delta x=\sqrt{ }\left(1-\alpha x^{2}+x^{4}\right) ; \text {; }
$$

diese Form geht sogleich in die gewöhnliche über, wenn man $\alpha=k+\frac{1}{k}$ nimmt und $x \sqrt{ } / k$, resp. $t / k$, an die Stelle von $x$ resp. $t$ setzt. - Die Gleichung (1.) nach $t$ differenziirt, giebt:

$$
\frac{\partial^{2} x}{\partial t^{2}}=\frac{\partial \Delta(x)}{\partial t}=-\alpha x+2 x^{3}
$$

Es sei $\boldsymbol{F}$ irgend eine Function von $x$, so ist

$$
\begin{aligned}
\frac{\partial^{2} F}{\partial t^{2}} & =\frac{\partial^{2} F}{\partial x^{2}}\left(\frac{\partial x}{\partial t}\right)^{2}+\frac{\partial F}{\partial x} \frac{\partial^{2} x}{\partial t^{2}}, \text { d. h. } \\
\text { (2.) } \frac{\partial^{2} F}{\partial t^{2}} & =\left(1-\alpha x^{2}+x^{4}\right) \frac{\partial^{2} F}{\partial x^{2}}+\left(-\alpha x+2 x^{3}\right) \frac{\partial F}{\partial x} .
\end{aligned}
$$

Wenn $\boldsymbol{F}$ eine ganze Function von $\boldsymbol{x}$ ist, so zeigt diese Formel (2.), dafs $\frac{\partial^{2} F}{\partial t^{2}}$ ebenfalls eine ganze Function von $x$ sein wird, deren Grad um 2 Einheiten höher ist, als der von $\boldsymbol{F}$; und zwar wird diese neue ganze Function mit $F$ zugleich gerade oder ungerade sein. Nun ist $\frac{\partial^{2} x}{\partial t^{2}}$ eine ganze Function von $x$, also auch jeder gerade Differenzialquotient von $x$ nach $t$ einer ganzen Function von $x$ gleich, und zwar wird dieselbe nur ungerade Potenzen von $x$ enthalten; jeder ungerade Differenzialquotient von $x$ nach $t$ ist dagegen gleich einem Producte aus einer ganzen und geraden Function von $x$ in $\boldsymbol{\Delta}(\boldsymbol{x})$. Natürlich gelten dieselben Beziehungen zwischen $y$ und $u$, wenn $\varphi(u)=y$ gesetzt wird, so dafs $\frac{\partial^{2} \mu}{\partial u^{2 \mu}}=\boldsymbol{P}, \frac{\partial^{2 \mu+1} y}{\partial u^{2 \mu+1}}=\boldsymbol{Q} \Delta(y)$, wo $\boldsymbol{P}$ eine ungerade, $\boldsymbol{Q}$ eine gerade ganze Function von $\boldsymbol{y}$ ist.

Man kann nach dem Taylorschen Satze folgende Entwickelung annehmen

$$
\varphi(t+u)=\alpha y+\beta \frac{\partial y}{\partial u}+\gamma \frac{\partial^{2} y}{\partial u^{2}}+\delta \frac{\partial^{3} y}{\partial u^{3}}+\text { etc. },
$$


deren Coëfficienten nur von $t \mathrm{~d}$. h. von $x$ abhängen. Zufolge des so eben über die Differenzialquotienten von $y$ nach $u$ Bemerkten läfst sich dieser Entwickelung die Form geben:

$$
\varphi(\boldsymbol{t}+\boldsymbol{u})=\boldsymbol{U}+\boldsymbol{V} \boldsymbol{A}(\boldsymbol{y}) .
$$

$\boldsymbol{U}$ und $\boldsymbol{V}$ sind Reihen, welche nach ganzen Potenzen von $\boldsymbol{y}$ fortlaufen, $\boldsymbol{U}$ enthält nur ungerade, $V$ nur gerade Potenzen von $y$. Offenbar ist dann auch

$$
\begin{aligned}
\varphi(\boldsymbol{t}-\boldsymbol{u}) & =-\boldsymbol{U}+\boldsymbol{V} \boldsymbol{A}(\boldsymbol{y}), \text { also } \\
\varphi(\boldsymbol{t}+\boldsymbol{u})+\varphi(\boldsymbol{t}-\boldsymbol{u}) & =2 \boldsymbol{V} \boldsymbol{\Delta}(\boldsymbol{y}) .
\end{aligned}
$$

Es sei der Kürze halber $\varphi(t+u)+\varphi(t-u)=2 \boldsymbol{W}$, so kann man selzen:

$$
\text { (3.) } \quad W=\sum_{\mu=0}^{\mu=\infty} C_{\mu} y^{2 \mu} \Delta(y) \text {. }
$$

Hieraus folgt, wenn man auf beiden Seiten nach $u$ differenziirt

$$
\begin{aligned}
\frac{\partial W}{\partial u} & =\Sigma C_{\mu}\left\{2 \mu y^{2 \mu-1}\left(1-\alpha y^{2}+y^{4}\right)+y^{2 \mu}\left(-\alpha y+2 y^{3}\right)\right\} \\
& =\Sigma 2 \mu C_{\mu} y^{2 \mu-1}-\Sigma(2 \mu+1) \alpha C_{\mu} y^{2 \mu+1}+\Sigma(2 \mu+2) C_{\mu} y^{2 \mu+3} \\
& =\Sigma\left\{(2 \mu+2) C_{\mu+1}-(2 \mu+1) \alpha C_{\mu}+2 \mu C_{\mu-1}\right\} y^{2 \mu+1} .
\end{aligned}
$$

Wird noch einmal nach $u$ differenziirt, so kommt

$$
\frac{\partial^{2} W}{\partial u^{2}}=\Sigma\left\{(2 \mu+1)(2 \mu+2) C_{\mu+1}-(2 \mu+1)^{2} \alpha C_{\mu}+2 \mu(2 \mu+1) C_{\mu-1}\right\} y^{2 \mu} \Delta(y) \text {. }
$$

Von der andern Seite ist offenbar

$$
\frac{\partial^{2} W}{\partial u^{2}}=\frac{\partial^{2} W}{\partial t^{2}}, \quad \text { also } \quad \frac{\partial^{2} W}{\partial u^{2}}=\Sigma \frac{\partial^{2} C_{\mu}}{\partial t^{2}} y^{2 \mu} \Delta(y)
$$

Die Vergleichung dieser beiden für $\frac{\partial^{2} W^{r}}{\partial \iota^{2}}$ erhaltenen Reihen liefert die Recursionsformel

$$
\text { (4.) } \quad(2 \mu+1)(2 \mu+2) \boldsymbol{C}_{\mu+1}=(2 \mu+1)^{2} \alpha \boldsymbol{C}_{\mu}-2 \mu(2 \mu+1) \boldsymbol{C}_{\mu-1}+\frac{\partial^{2} \boldsymbol{C}}{\partial t^{2}} \text {. }
$$

Nach dieser Formel kann der zweite Coëfficient der. Reihe $\boldsymbol{W}$ aus dem ersten und jeder folgende aus den beiden vorhergehenden berechnet werden. Um den ersten Coëfficienten $\boldsymbol{C}_{0}$ zu finden, setze man $\boldsymbol{u}=0$; dann verschwinden alle Glieder der Reihe für $W$ bis auf das erste, $\Delta(y)$ wird $=1$ und $W=$ $\frac{1}{2}(\varphi(t)+\varphi(t))=x$, also erhält man

$$
\boldsymbol{C}_{0}=\boldsymbol{x} \text {. }
$$

Wird nun endlich in (2.) $\boldsymbol{F}=x^{2 \mu+1}$ gesetzt, so kommt

$$
\begin{aligned}
\frac{\partial^{2}\left(x^{2 \mu+1}\right)}{\partial t^{2}} & =2 \mu(2 \mu+1) x^{2 \mu-1}\left(1-\alpha x^{2}+x^{4}\right)+(2 \mu+1) x^{2 \mu}\left(-\alpha x+2 x^{3}\right) \\
& =2 \mu(2 \mu+1) x^{2 \mu-1}-(2 \mu+1)^{2} \alpha x^{2 \mu+1}+(2 \mu+1)(2 \mu+2) x^{2 \mu+3},
\end{aligned}
$$

also

$$
(2 \mu+1)(2 \mu+2) x^{2 \mu+3}=(2 \mu+1)^{2} \alpha x^{2 \mu+1}-2 \mu(2 \mu+1) x^{2 \mu-1}+\frac{\partial^{2}\left(x^{2 \mu+1}\right)}{\partial t^{2}}
$$


Diese Formel, mit (4.) verglichen, liefert, wenn man bedenkt, dafs $C_{0}=x$ ist,

$$
C_{1}=x^{3}, \quad C_{2}=x^{5}, \quad \text { u. s. w. }
$$

und allgemein

$$
\boldsymbol{C}_{\mu}=\boldsymbol{x}^{2 \mu+1}
$$

also hat man

$$
\begin{gathered}
W=x \Delta(y)+x^{3} y^{2} \Delta(y)+x^{5} y^{4} \Delta(y)+\text { etc. }=\frac{x \Delta(y)}{1-x^{2} y^{2}}, \text { d. h. } \\
\text { (6.) } \quad \varphi(t+u)+\varphi(t-u)=\frac{2 x \Delta(y)}{1-x^{2} y^{2}} .
\end{gathered}
$$

Vertauscht man $t$ und $u$, so bleibt $\varphi(t+u)$ unverändert und $\varphi(t-u)$ geht in $\varphi(\boldsymbol{u}-\boldsymbol{t})=-\varphi(\boldsymbol{t}-\boldsymbol{u})$ über, also erhält man noch

$$
\text { (7.) } \varphi(t+u)-\varphi(t-u)=\frac{2 y \Delta(x)}{1-x^{2} \gamma^{2}} \text {. }
$$

Addirt und subtrahirt man (6.) und (7.), so kommt

$$
\text { (8.) }\left\{\begin{array}{l}
\varphi(\boldsymbol{t}+\boldsymbol{u})=\frac{x \Delta(y)+y \Delta(x)}{1-x^{2} y^{2}} \\
\varphi(\boldsymbol{t}-\boldsymbol{u})=\frac{x \Delta(y)-y \Delta(x)}{1-x^{2} y^{2}}
\end{array}\right.
$$

Dies sind die verlangten Formeln, welche die gewöhnliche Form annehmen, wenn man, wie schon bemerkt, $\varphi(t)=\sqrt{ } k \cdot \sin$ am $\left(\frac{t}{\sqrt{ } k}\right)$ setzt. 